\title{
Probing the pre-BBN universe with gravitational waves from cosmic strings
}

\author{
Yanou Cui, ${ }^{a}$ Marek Lewicki, ${ }^{b, c}$ David E. Morrissey ${ }^{d}$ and James D. Wells ${ }^{e, f}$ \\ ${ }^{a}$ Department of Physics and Astronomy, University of California, \\ 900 University Avenue, Riverside, CA 92521, U.S.A. \\ ${ }^{b}$ Kings College London, \\ Strand, London, WC2R 2LS, U.K. \\ ${ }^{c}$ Faculty of Physics, University of Warsaw, \\ ul. Pasteura 5, 02-093 Warsaw, Poland \\ ${ }^{d}$ TRIUMF, \\ 4004 Wesbrook Mall, Vancouver, BC V6T 2A3, Canada \\ ${ }^{e}$ Leinweber Center for Theoretical Physics, University of Michigan, \\ 450 Church Street, Ann Arbor, $M I$ 48109, U.S.A. \\ ${ }^{f}$ Theory Group, Deutsches Elektronen-Synchrotron DESY, \\ D-22603 Hamburg, Germany \\ E-mail: yanou.cui@ucr.edu, marek.lewicki@kcl.ac.uk, dmorri@triumf.ca, \\ jwells@umich.edu
}

Abstract: Many motivated extensions of the Standard Model predict the existence of cosmic strings. Gravitational waves originating from the dynamics of the resulting cosmic string network have the ability to probe many otherwise inaccessible properties of the early universe. In this study we show how the spectrum of gravitational waves from a cosmic string network can be used to test the equation of state of the early universe prior to Big Bang Nucleosynthesis (BBN). We also demonstrate that current and planned gravitational wave detectors such as LIGO, LISA, DECIGO/BBO, and ET/CE have the potential to detect signals of a non-standard pre-BBN equation of state and evolution of the early universe (e.g., early non-standard matter domination or kination domination) or new degrees of freedom active in the early universe beyond the sensitivity of terrestrial collider experiments and cosmic microwave background measurements.

KEywords: Beyond Standard Model, Cosmology of Theories beyond the SM

ARXIV EPRINT: 1808.08968 


\section{Contents}

1 Introduction 1

2 GW spectrum of a cosmic string network 3

2.1 Cosmic string scaling and loop production 4

2.2 Derivation of the GW frequency spectrum 5

2.3 Connecting GW frequencies to loop formation and emission times $\quad 7$

3 Mapping the early universe with cosmic string GWs 9

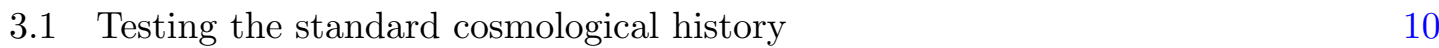

$\begin{array}{lll}3.2 & \text { Probing new degrees of freedom } & 12\end{array}$

$\begin{array}{lll}3.3 & \text { Probing new phases of cosmological evolution } & 14\end{array}$

4 Detection challenges and ways to overcome them $\quad \mathbf{1 5}$

4.1 Astrophysical backgrounds 16

$\begin{array}{ll}4.2 & \text { Distinguishing cosmic strings from other new phenomena }\end{array}$

$\begin{array}{lll}4.3 & \text { Sensitivity to the loop size parameter } \alpha & 18\end{array}$

$\begin{array}{llr}5 & \text { Conclusions } & 19\end{array}$

\section{Introduction}

Remarkable progress has been made in understanding the universe through detailed observations of the electromagnetic radiation emitted by the cosmos. These measurements, spanning a range of frequencies from radio to gamma-ray [1], have led to the $\Lambda \mathrm{CDM}$ model of cosmology in which the universe is currently dominated by dark energy and cold dark matter with smaller components of baryonic matter and radiation [2].

Extrapolating the $\Lambda \mathrm{CDM}$ model back in time suggests that the very early universe was dominated by radiation in the form of photons and other relativistic particles. This extrapolation is strongly supported by measurements of the cosmic microwave background (CMB), corresponding to the photons that escaped after recombination when the radiation temperature was about $0.3 \mathrm{eV}$ [2]. The success of Big Bang Nucleosynthesis (BBN) in predicting primordial light element abundances gives additional convincing evidence for early radiation domination $(\mathrm{RD})$ up to temperatures close to $T \simeq 5 \mathrm{MeV}$ [3-5]. Going even further back, the observed flatness and uniformity of the cosmos and the spectrum of density perturbations suggest that this radiation era was preceded by a period of rapid expansion such as inflation [6-9].

Very little is known empirically about the state of the universe between the end of inflation and the start of BBN [10]. A minimal assumption is that inflation was followed 
by reheating to a very hot radiation phase with temperature $T \gg \mathrm{TeV}$, which then cooled adiabatically until giving way to the recent matter and dark energy phases. We refer to this scenario, with only radiation domination (RD) over many orders of magnitude in temperature between reheating and the matter epoch, as the standard cosmology [11]. While this assumption is made frequently (and often implicitly), it has not been tested directly. Furthermore, non-standard cosmological scenarios with an extended period of domination by something other than radiation between inflation and BBN have strong motivation from many perspectives, including dark matter, axions, string compactification, reheating, and baryogenesis [10, 12-26]. Testing the paradigm of pre-BBN cosmology is therefore of great significance in advancing our understanding of the universe.

Gravitational waves (GWs) may provide a means of looking back in time beyond the BBN epoch and probing the universe in its very early stages [10,27-29]. The observation of binary mergers by the LIGO/Virgo collaboration has already given further support to the $\Lambda \mathrm{CDM}$ cosmology [30], although - and this is important to the motivation of this work the GWs observed were created only relatively recently. Opportunity to look even further back in time with GWs arises because, in contrast to photons, GWs free-stream throughout the entire history of the cosmos. Indeed, GWs emitted as far back as inflation could potentially be detected by LIGO/Virgo [31] or proposed future detectors such as LISA [32], BBO/DECIGO [33], the Einstein Telescope (ET) [34, 35], and Cosmic Explorer (CE) [36].

A stable and predictable source of primordial GWs is needed if they are to be used to test very early cosmology. Two promising and well-motivated potential sources are cosmic strings [37-40] and primordial inflation [41-43]. The application of inflationary GWs to probe the expansion history of the universe was studied in refs. [44-47] and for non-standard histories in refs. [48-51]. However, current limits from CMB isotropy typically push the inflationary stochastic GW spectra below the sensitivity of current and next generation detectors [52-54] (although see ref. [55]). Thus, we focus on GWs from cosmic strings in this work.

Cosmic strings are approximately one-dimensional objects of macroscopic length that arise nearly generically in theories of physics beyond the Standard Model (SM). Specific examples include topologically-stable field configurations in theories with a spontaneously broken $\mathrm{U}(1)$ symmetry $[56,57]$, as well as fundamental or composite strings in superstring theory [58-62]. Their macroscopic properties are mostly characterized by their tension (energy per unit length) $\mu$, which is typically on the order of the square of the energy scale of new physics that gives rise to them, and directly constrained by CMB measurements to $G \mu<1.1 \times 10^{-7}$ [63], where $G$ is Newton's constant.

Cosmic strings emit gravitational radiation as part of their cosmological evolution [6467]. After formation, cosmic strings are expected to quickly reach a scaling regime in which their net energy density tracks the total cosmological energy density with a relative fraction $G \mu$ [68-70]. This regime consists of a small number of Hubble-length long strings and a collection of many closed string loops. As the universe expands, the long strings intersect and intercommute to form new loops, while the existing loops oscillate and emit radiation, including GWs. This continual transfer of energy from long strings to loops to radiation is essential for the string network density to track the total energy density of the universe, 
rather than becoming dominant like other topological defects such as monopoles [71, 72] and domain walls [73]. In particular, the presence of cosmic strings with small $G \mu \ll 1$ need not disrupt the standard cosmology.

For many classes of cosmic strings, the dominant radiation emission is in the form of GWs. This is true for ideal Nambu-Goto strings, many types of cosmic strings emerging from superstring theory, and possibly those created by local U(1) symmetry breaking [74, 75] (although see refs. [76-79] for arguments that local strings emit mainly massive vector and Higgs quanta instead). In contrast, cosmic strings derived from global symmetry breaking are expected to radiate mainly to light Goldstone quanta [80-84], with much weaker emission to GWs. We focus on cosmic strings that radiate significantly to GWs through loop formation and emission in this work.

The GW frequency spectrum from a cosmic string network is sensitive to the evolution of the cosmos when the GWs were emitted. In any given frequency band observed today, the dominant contribution to the signal comes from loops emitting GWs at a specific time in the early universe $[27,28,38]$. As a result of this frequency-time relation, we show that the cosmological equation of state leaves a distinct imprint on the frequency spectrum of GWs from cosmic strings. Moreover, the portion of the spectrum from loops formed and emitting during RD has a distinctive nearly flat plateau with a substantial amplitude over many decades in frequency. Measuring the GW signal from a cosmic string network over a range of frequencies could therefore provide a unique picture of the very early universe that could potentially expand back before the era of BBN.

The outline of this paper is as follows. After this introduction, we review cosmic string scaling and derive the GW frequency spectrum from a string network in section 2. We also exhibit the relationship between the GW spectrum and the loop emission rates and formation times, and apply these to the concurrent background cosmology. In section 3 we show how this relationship together with the anticipated sensitivities of current and planned GW detectors can be used to test the standard cosmological scenario as well as deviations from it, including large numbers of additional (massive) degrees of freedom and modified equations of state. Some of the challenges to detecting these GW signals and identifying them as coming from cosmic strings, and ways to overcome them, are discussed in section 4. Finally, section 5 is reserved for our conclusions.

The results in this paper expand upon those of our previous study in ref. [28]. Relative to the work, we present in great detail the time-frequency connection of cosmic string GWs and its relation to the background cosmology. We also expand significantly on the experimental sensitivity of GW probes to new degrees of freedom active during early universe with presence of cosmic string dynamics, and extend our study of standard and modified cosmological histories.

\section{GW spectrum of a cosmic string network}

In this section we derive the GW frequency spectrum from a cosmic string network. We assume a network of ideal Nambu-Goto cosmic strings with unit reconnection probability and dominant energy loss through loop formation and emission of gravitational radiation. 


\subsection{Cosmic string scaling and loop production}

Cosmic string scaling is achieved through a balance of the slow $a^{-2}$ dilution of the horizonlength long-string density and the transfer of energy out of the long-string network by the production of closed string loops [40]. These loops oscillate, emit energy in gravitational radiation, and eventually decay away. To compute the GW spectrum from these processes, estimates are needed for the sizes and rates of the loops formed by the long string network.

Recent cosmic string simulations find that a fraction of about $10 \%$ of the energy transferred by the long strings to loops is in the form of relatively large loops, with the remaining $90 \%$ going to highly boosted small loops [85-90]. The large loops give the dominant contribution to the GW signal and we focus exclusively on them, since the relativistic small loops lose most of their energy to simple redshifting. Large loops are formed with a characteristic initial length $l_{i}$ that tracks the time $t_{i}$ of formation,

$$
l_{i}=\alpha t_{i},
$$

where $\alpha$ is an approximately constant loop size parameter [85-91]. We make the simplifying assumption of monochromatic (large) loop formation with $\alpha=0.1$, which gives a good reproduction of the loop size distribution of refs. [90, 91]. We comment on the impact of modifying the value of $\alpha$ in section 4.3.

The formation rate of (large) loops by a scaling string network is also needed to compute the GW spectrum. For this, we use the velocity-dependent one-scale (VOS) model to describe the properties of the long string network in the scaling regime [92-96], and we match the rate of energy release by the long string network needed to maintain scaling with the rate of energy going to loops [40]. The VOS model describes the long string network in terms of a characteristic length (as a fraction of the horizon) $\xi$ and a mean string velocity $\bar{v}$, and is found to give a good analytic description of the network properties during scaling. Let us emphasize, however, that we only use the VOS model to describe the long string network; we base the structure of the loops on the results of direct simulations [90, 91].

Consider a scaling network evolving in a cosmological background driven by a dominant source of energy density that dilutes according to

$$
\rho \propto a^{-n} .
$$

This implies $a(t) \propto t^{2 / n}$, with $n=3,4$ giving the familiar cases of matter and radiation domination. Within such a background, the VOS model describes the long string network in terms of a characteristic string velocity $\bar{v}$ and length parameter $\xi$ given by [92-94]

$$
\xi=\frac{n}{2} \sqrt{\frac{k(\bar{v})[k(\bar{v})+\bar{c}]}{2(n-2)}}, \quad \bar{v}=\sqrt{\frac{n}{2} \frac{k(\bar{v})}{[k(\bar{v})+\bar{c}]}\left(1-\frac{2}{n}\right)},
$$

where $\bar{c}$ is a loop chopping efficiency parameter and $k(\bar{v})$ is a function of $\bar{v}$ to be determined. We fix $\bar{c}=0.23$ based on numerical simulations [94], and we use the ansatz of ref. [94] for the function $k(\bar{v})$ :

$$
k(\bar{v})=\frac{2 \sqrt{2}}{\pi}\left(1-\bar{v}^{2}\right)\left(1+2 \sqrt{2} \bar{v}^{3}\right) \frac{1-8 \bar{v}^{6}}{1+8 \bar{v}^{6}} .
$$




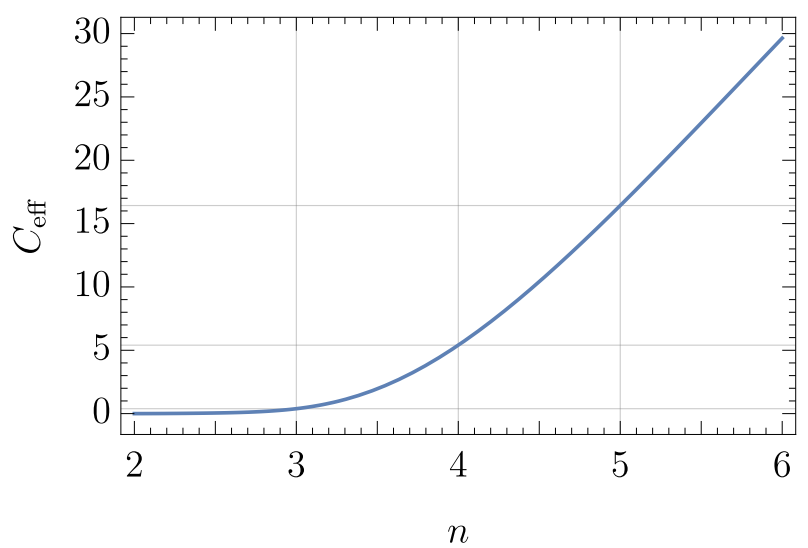

Figure 1. Dependence of the loop emission factor $C_{\text {eff }}$ derived from the VOS model on the cosmological energy density redshift parameter $n$ defined in eq. (2.2).

In terms of $\xi$ and $\bar{v}$, the energy density $\rho_{L}$ of the long string network is

$$
\rho_{L}=\frac{\mu}{(\xi t)^{2}}
$$

while the rate of energy loss needed to maintain scaling is

$$
\frac{d \rho_{L}}{d t}=\bar{c} \bar{v} \frac{\rho_{L}}{(\xi t)} .
$$

To estimate the loop formation rate, we identify the energy loss rate of eq. (2.6) with the rate of energy transferred to loops. If large loops of initial size $l_{i}=\alpha t_{i}$ and Lorentz boost $\gamma$ make up a fraction $\mathcal{F}_{\alpha}$ of the energy released by long strings, their formation rate is

$$
\frac{d n_{\alpha}}{d t}=\mathcal{F}_{\alpha} \frac{C_{\text {eff }}}{\alpha} t^{-4}
$$

with

$$
C_{\mathrm{eff}}=\frac{\tilde{c}}{\gamma} \bar{v} \xi^{-3} .
$$

Recent simulations find $\alpha \simeq 0.1, \mathcal{F}_{\alpha} \simeq 0.1$, and $\gamma \simeq \sqrt{2}$, and we use these as default values in the analysis to follow [90, 91].

In figure 1 we show the result for $C_{\text {eff }}$ as a function of the background cosmology scaling factor over the range $n \in[2,6]$. We find $C_{\text {eff }}=0.39$ and 5.4 during matter $(n=3)$ and radiation $(n=4)$ domination, respectively, which compare well with $C_{\text {eff }}=0.5$ and 5.7 found in detailed lattice simulations [89-91]. The method used here can be applied to other cosmological backgrounds, and in particular we note that $C_{\text {eff }}(n=6) \simeq 30.4$.

\subsection{Derivation of the GW frequency spectrum}

After formation, loops are found to emit energy in the form of gravitational radiation at a constant rate

$$
\frac{d E}{d t}=-\Gamma G \mu^{2}
$$


where $\Gamma \approx 50$ is a dimensionless constant $[64,66,90,91,97]$. Thus, the length of a loop with initial size $l_{i}=\alpha t_{i}$ decreases as

$$
l(t)=\alpha t_{i}-\Gamma G \mu\left(t-t_{i}\right)
$$

until the loop disappears completely. The total energy loss from a loop can be decomposed into a set of normal-mode oscillations at frequencies $\tilde{f}_{k}=2 k / l$, where $k=1,2,3 \ldots$ is the mode number. The relative emission rate per mode is found to scale with $k^{-4 / 3}$ and is given by $[90,91]$

$$
\Gamma^{(k)}=\frac{\Gamma k^{-\frac{4}{3}}}{\sum_{m=1}^{\infty} m^{-\frac{4}{3}}} .
$$

Note that $\sum_{m=1}^{\infty} m^{-\frac{4}{3}} \simeq 3.60$ and $\sum_{k} \Gamma^{(k)}=\Gamma$. After emission at time $\tilde{t}$ the frequency of the GW redshifts, so the frequency observed today is $f=\left[a(\tilde{t}) / a\left(t_{0}\right)\right] \tilde{f}$.

Combining the GW emission rate per loop of eq. (2.9), the emitted frequencies of eq. (2.11), and the rate of (large $\alpha=0.1$ ) loop formation of eq. (2.7), we can compute the relic GW background from a cosmic string network. It is conventional to express this background in terms of

$$
\Omega_{\mathrm{GW}}=\frac{f}{\rho_{c}} \frac{d \rho_{\mathrm{GW}}}{d f}
$$

where $\rho_{\mathrm{GW}}$ is the energy density of GWs, $f$ is the frequency today, and $\rho_{c}=3 H_{0}^{2} / 8 \pi G$ is the critical density. Summing over all mode contributions,

$$
\Omega_{\mathrm{GW}}(f)=\sum_{k} \Omega_{\mathrm{GW}}^{(k)}(f),
$$

with

$$
\Omega_{\mathrm{GW}}^{(k)}(f)=\frac{1}{\rho_{c}} \frac{2 k}{f} \frac{\mathcal{F}_{\alpha} \Gamma^{(k)} G \mu^{2}}{\alpha(\alpha+\Gamma G \mu)} \int_{t_{F}}^{t_{0}} d \tilde{t} \frac{C_{\mathrm{eff}}\left(t_{i}^{(k)}\right)}{t_{i}^{(k) 4}}\left[\frac{a(\tilde{t})}{a\left(t_{0}\right)}\right]^{5}\left[\frac{a\left(t_{i}^{(k)}\right)}{a(\tilde{t})}\right]^{3} \Theta\left(t_{i}^{(k)}-t_{F}\right)
$$

where the integral runs over the GW emission time $\tilde{t}$, and

$$
t_{i}^{(k)}(\tilde{t}, f)=\frac{1}{\alpha+\Gamma G \mu}\left[\frac{2 k}{f} \frac{a(\tilde{t})}{a\left(t_{0}\right)}+\Gamma G \mu \tilde{t}\right] .
$$

is the formation time of loops contributing with mode number $k$, and $t_{F}$ is the time at which the cosmic string network reached scaling, shortly after the formation of the network. Note that the sum in eq. (2.13) is easily evaluated because $\Omega_{\mathrm{GW}}^{(k)}(f)=\frac{\Gamma^{(k)}}{\Gamma^{(1)}} \Omega_{\mathrm{GW}}^{(1)}(f / k)=$ $k^{-4 / 3} \Omega_{\mathrm{GW}}^{(1)}(f / k)$.

We show in figure 2 the GW spectrum from a cosmic string network with $\alpha=0.1$, $G \mu=10^{-11}, 10^{-13}, 10^{-15}, 10^{-17}$, assuming standard cosmological evolution. Also shown are the current and future sensitivities of LIGO [98-101], and the projected sensitivities of LISA [102], DECIGO/BBO [33], Einstein Telescope (ET) [34, 35], and Cosmic Explorer (CE) [36]. The solid triangle in the upper left of the plot indicates the current limit from the European Pulsar Timing Array (EPTA) [103], and the expected sensitivity of the 


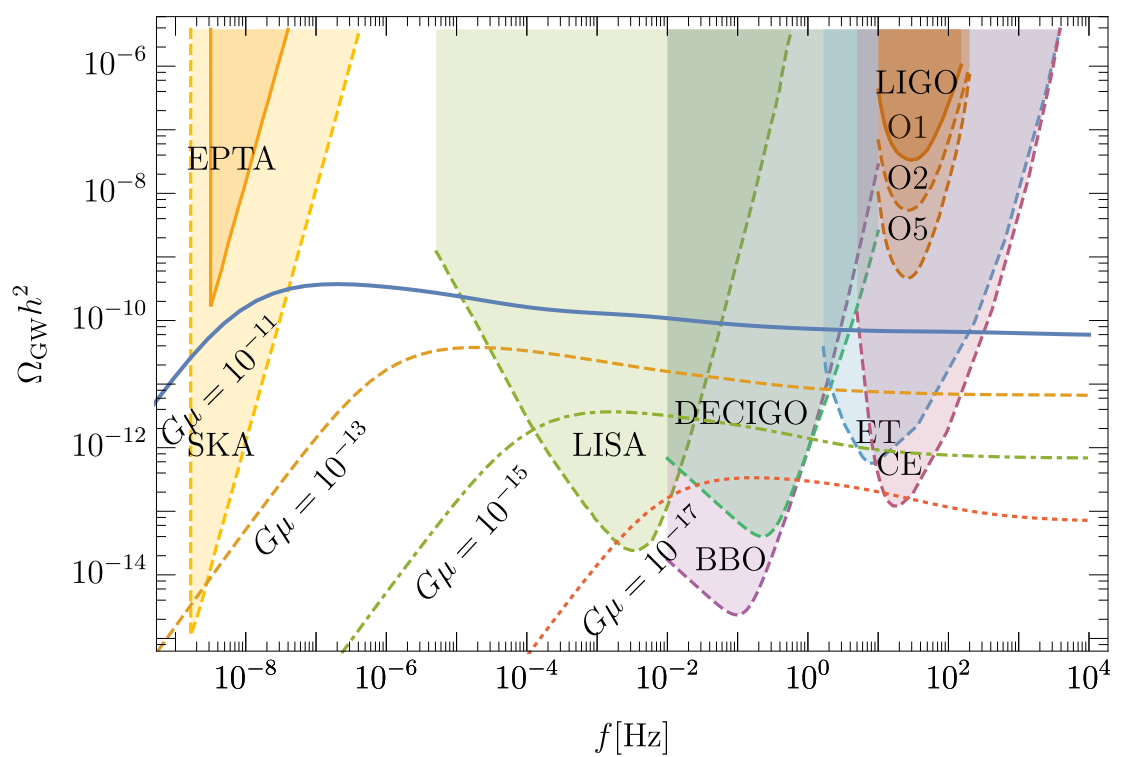

Figure 2. Gravitational wave spectrum from a cosmic string network with $\alpha=0.1$ and $G \mu=$ $10^{-11}, 10^{-13}, 10^{-15}, 10^{-17}$. Also shown are the current sensitivities of LIGO and EPTA (solid bounded regions), and the projected future sensitivities of LISA, DECIGO/BBO, ET/CE, and SKA (dash bounded regions).

future Square Kilometre Array (SKA) [104]. We see that the strongest current bound on these GW spectra comes from EPTA and implies $G \mu \lesssim 2 \times 10^{-11}$. Other recent estimates of the GW spectrum from a scaling cosmic string network relative to current and future searches includes refs. $[90,105,106]$.

\subsection{Connecting GW frequencies to loop formation and emission times}

The GW spectra shown in figure 2 all share a characteristic shape, with a dropoff at lower frequencies and a flattening at higher ones. This shape is related to the cosmological background evolution when the loops contributing to a given frequency were formed and emitted GWs [27]. In this section, we connect the GW frequency seen today to the time at which the dominant contribution to that frequency was emitted by the string network. Later, we show how this connection can be used to test the evolution of the very early universe.

We begin with a simple analytic estimate of the frequency-time connection. (See also ref. [107].) For this, it is sufficient to focus exclusively on the $k=1$ mode which we find to be the dominant one in the cases of interest. We also set $t_{F} \rightarrow 0$ for now, and return to nonzero values later on. The expression of eq. (2.14) involves an integral over the GW emission time $\tilde{t}$, with the contribution to the signal over the time interval $(\tilde{t}, \tilde{t}+d \tilde{t})$ proportional to

$$
d \Omega_{\mathrm{GW}}(f) \propto d \tilde{t} \times \mathcal{I}(\tilde{t}, f) \equiv d \tilde{t} \times \frac{1}{f} t_{i}^{-4}\left(\frac{a_{i}}{\tilde{a}}\right)^{3}\left(\frac{\tilde{a}}{a_{0}}\right)^{5},
$$

with $a_{i}=a\left(t_{i}\right), \tilde{a}=a(\tilde{t})$, and $t_{i}$ given by eq. (2.15) with $k=1$. The $\tilde{t}$ and $f$ dependence of the function $\mathcal{I}(\tilde{t}, f)$ is approximately power-law, and depends on the dominant cosmo- 
logical background energy source at times $\tilde{t}$ and $t_{i}$. Let us define the time $\tilde{t}_{M}(f)$ to be the value of $\tilde{t}$ when the two terms in $t_{i}$ are equal in size:

$$
\frac{2}{f} \frac{a\left(\tilde{t}_{M}\right)}{a_{0}}=\Gamma G \mu \tilde{t}_{M}
$$

If the background energy redshifts as $\rho\left(t_{i}\right) \propto a^{-m}$ at time $t_{i}$ and $\rho(\tilde{t}) \propto a^{-n}$ at time $\tilde{t}$, the approximate $\tilde{t}$ and $f$ dependence of $\mathcal{I}(\tilde{t}, f)$ is

$$
\mathcal{I}(\tilde{t}, f) \propto \begin{cases}f^{(3 m-6) / m} \tilde{t}^{(2 / n)(6-4 m) / m+4 / n} ; & \tilde{t}<\tilde{t}_{M} \\ f^{-1} \tilde{t}^{(6-4 m) / m+4 / n} ; & \tilde{t} \geq \tilde{t}_{M}\end{cases}
$$

Integrating this power-law form is straightforward, with the indefinite integral scaling according to

$$
\int d \tilde{t} \mathcal{I}(\tilde{t}, f) \propto \tilde{t}^{p}
$$

with

$$
p= \begin{cases}p_{1}=1+(2 / n)(6-4 m) / m+4 / n ; & \tilde{t}<\tilde{t}_{M} \\ p_{2}=1+(6-4 m) / m+4 / n ; & \tilde{t} \geq \tilde{t}_{M}\end{cases}
$$

To evaluate eq. (2.14) in this approximation, we divide the integral over $\tilde{t}$ into nonoverlapping regions with distinct $(m, n)$ values and sum the piecewise contributions of the form of eqs. (2.19) and (2.20). The power $p_{1}$ is positive for the ranges of interest $m, n \in(2,6]$, implying that the contribution to the definite integral from $\tilde{t}<\tilde{t}_{M}$ is dominated by $\tilde{t} \sim \min \left\{\tilde{t}_{M}, t_{0}\right\}$. If $\tilde{t}_{M}<t_{0}$ and $p_{2}<0$, which is true for most cases of interest in this work, the contribution from $\tilde{t}>\tilde{t}_{M}$ is also dominated by $\tilde{t} \sim \tilde{t}_{M}$ and has the same parametric size as that from $\tilde{t}<\tilde{t}_{M}$. In contrast, for $p_{2}>0$ the integral is dominated by the largest value of $\tilde{t}$ in the corresponding $(m, n)$ region.

The result of eq. (2.18) can also be used to derive the approximate frequency dependence of $\Omega_{\mathrm{GW}}(f)$. We find

$$
\Omega_{\mathrm{GW}}(f) \propto \begin{cases}f^{(3 m-6) / m} ; & \tilde{t}_{M} \geq t_{0} \\ f^{2(m n-m-3 n) / m(n-2)} ; & \tilde{t}_{M}<t_{0}, p_{2}<0 \\ f^{-1} ; & \tilde{t}_{M}<t_{0}, p_{2} \geq 0\end{cases}
$$

where $(m, n)$ refer to the cosmological scalings specifically at $t_{i}(\tilde{t}, f)$ and $\tilde{t}$ for $\tilde{t}=$ $\min \left\{\tilde{t}_{M}(f), t_{0}\right\}$. For loop formation and GW emission in the radiation era, $(m, n) \simeq(4,4)$ and $\Omega_{\mathrm{GW}} \propto f^{0}$, corresponding to the flat plateaus seen at higher frequencies in figure 2 . For loop formation in the radiation era and GW emission in the matter era, $(m, n)=(4,3)$ giving $\Omega_{\mathrm{GW}} \propto f^{-1 / 2}$, which coincides with the decrease seen in figure 2 prior to the flat plateau. The rising spectrum at low frequencies corresponds to $\tilde{t}_{M}(f)>t_{0}$ (and $t_{i}<t_{\text {eq }}$ unless $f$ is very small), for which the dominant emission occurs around $\tilde{t} \sim t_{0}$ implying $\Omega_{\mathrm{GW}}(f) \propto f^{3 / 2}$.

It is also instructive to study the relative contributions to the spectrum from GW emission during the radiation and matter eras and the effect of finite $t_{F}$. These features are 


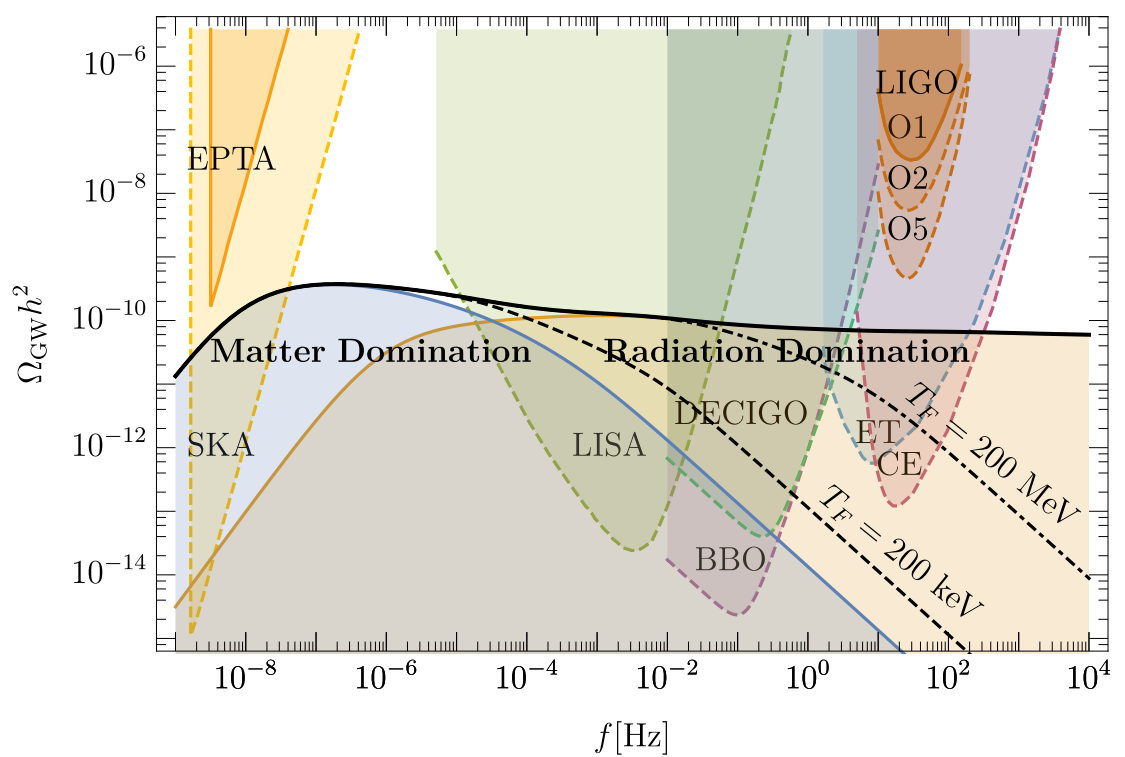

Figure 3. Gravitational wave spectrum from a cosmic string network with $G \mu=10^{-11}$ and $\alpha=10^{-1}$. The solid black line shows the full spectrum, while the blue shaded region shows the contribution from loops emitted during matter domination and the orange shaded region indicates the contribution from loops emitting in the radiation era. The black dashed lines show the effect on the spectrum if only those loops formed at temperatures below $T_{F}=200 \mathrm{keV}, 200 \mathrm{MeV}$ are included.

illustrated in figure 3, where we show the GW spectrum for $\alpha=0.1$ and $G \mu=10^{-11}$. The solid black line shows the full spectrum, the shaded blue region indicates the contribution from loop emission of GWs during the matter era $\left(t_{\text {eq }}<\tilde{t}<t_{0}\right)$, and the shaded orange shows the contribution from loop emission of GWs during the radiation era $\left(\tilde{t}<t_{\text {eq }}\right)$. Note that both the matter and radiation contributions in the figure are dominated by loops that were formed during the radiation era $\left(t_{i}\left(t_{0}\right)<t_{\mathrm{eq}}\right)$. The dashed (dot-dashed) line in this figure shows the effect of artificially increasing $t_{F}$ to the cosmological time corresponding to the radiation temperature $T_{F}=200 \mathrm{keV}(200 \mathrm{MeV})$. Finite $t_{F}$ imposes a lower cutoff on $t_{i}$ that modifies the spectrum when $t_{i}\left(\tilde{t}_{M}\right)<t_{F}$, implying that the spectrum falls off as $1 / f$ going to large frequency.

\section{Mapping the early universe with cosmic string GWs}

The analysis above shows the close connection between the frequency spectrum of GWs produced by a cosmic string network and the cosmological background when they were emitted. In this section we investigate how this property could be used to test history of the very early universe if a relic GW signal from a string network were to be detected. In particular, we demonstrate that the GW spectrum could be used to test the standard cosmological picture further back in time than the best current limits based on primordial Big Bang Nucleosynthesis (BBN). We also study how deviations from the standard picture would imprint themselves on the spectrum. 


\subsection{Testing the standard cosmological history}

Current observations provide strong evidence for the standard $\Lambda \mathrm{CDM}$ model of cosmology [2]. In this model, the very early universe (following a period of inflation or something similar) is dominated by radiation, followed by a period of matter domination, and very recently entering a phase of accelerated expansion driven by a constant dark energy. This evolution (after inflation) is encapsulated in the first Friedmann equation describing the expansion rate of the scale factor $a(t)$ :

$$
H^{2} \equiv\left(\frac{\dot{a}}{a}\right)^{2}=H_{0}^{2}\left[\Delta_{R}(a) \Omega_{R}\left(\frac{a}{a_{0}}\right)^{-4}+\Omega_{M}\left(\frac{a}{a_{0}}\right)^{-3}+\Omega_{\Lambda}\right],
$$

where $H_{0} \simeq 1.44 \times 10^{-42} \mathrm{GeV}$ is the expansion rate measured today, $\Omega_{R} \simeq 9.2 \times 10^{-5}$ for radiation, $\Omega_{M} \simeq 0.31$ for matter, and $\Omega_{\Lambda} \simeq 0.69$ for dark energy [108]. The correction factor

$$
\Delta_{R}(a)=\frac{g_{*}(a)}{g_{*}\left(a_{0}\right)}\left(\frac{g_{* S}\left(a_{0}\right)}{g_{* S}(a)}\right)^{4 / 3}
$$

accounts for the deviation from $T \propto a^{-1}$ dictated by entropy conservation, and depends on the effective number of energy density $\left(g_{*}\right)$ and entropy $\left(g_{* S}\right)$ degrees of freedom for which we use the SM parametrization in micrOMEGAs_3.6.9.2 [109].

An early period of domination by something other than radiation would show up in the GW frequency spectrum as a significant deviation from flatness. For given values of $G \mu$ and $\alpha$, the frequency $f_{\Delta}$ at which such a deviation would appear is determined by the cosmological time $t_{\Delta}$ when the (most recent) radiation era began. ${ }^{1}$ Based on eqs. $(2.18,2.21)$ and the analysis in section 2.3, the frequency spectrum is first modified significantly when the dominant emission time $\tilde{t}_{M}$ comes from loops created at $t_{i}^{(k=1)} \simeq t_{\Delta}$. This gives an approximate transition frequency $f_{\Delta}$ as the solution of

$$
t_{i}\left(\tilde{t}_{M}\left(f_{\Delta}\right)\right)=t_{\Delta}
$$

Approximating $a(t) \propto t^{1 / 2}$ during the radiation era, this gives

$$
\begin{aligned}
f_{\Delta} & \simeq \sqrt{\frac{8 z_{\mathrm{eq}}}{\alpha \Gamma G \mu}}\left(\frac{t_{\mathrm{eq}}}{t_{\Delta}}\right)^{1 / 2} t_{0}^{-1} \\
& \simeq \sqrt{\frac{8}{z_{\mathrm{eq}} \alpha \Gamma G \mu}}\left[\frac{g_{*}\left(T_{\Delta}\right)}{g_{*}\left(T_{0}\right)}\right]^{1 / 4}\left(\frac{T_{\Delta}}{T_{0}}\right) t_{0}^{-1}
\end{aligned}
$$

where $z_{\text {eq }} \simeq 3387$ is the redshift at matter-radiation equality, and $T_{0}=2.725 \mathrm{~K}$ is the temperature today. A more accurate dependence obtained by fitting to a full numerical calculation that properly accounts for variations in $g_{*}$ gives

$$
f_{\Delta}=\left(8.67 \times 10^{-3} \mathrm{~Hz}\right)\left(\frac{T_{\Delta}}{\mathrm{GeV}}\right)\left(\frac{0.1 \times 50 \times 10^{-11}}{\alpha \Gamma G \mu}\right)^{1 / 2}\left(\frac{g_{*}\left(T_{\Delta}\right)}{g_{*}\left(T_{0}\right)}\right)^{\frac{8}{6}}\left(\frac{g_{* S}\left(T_{0}\right)}{g_{* S}\left(T_{\Delta}\right)}\right)^{-\frac{7}{6}}
$$

which we find to be accurate to about $10 \%$.

\footnotetext{
${ }^{1}$ Equivalently, radiation domination occurred for $t_{\Delta}<t<t_{\text {eq }}$ with something else for $t<t_{\Delta}$.
} 


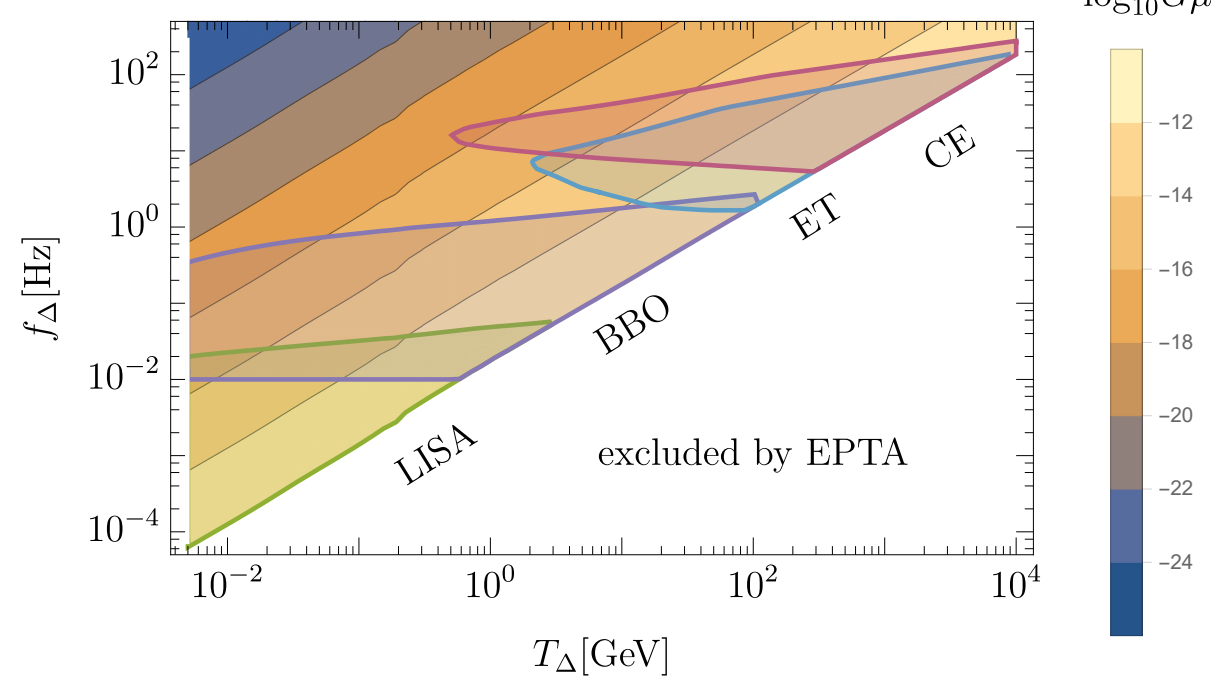

Figure 4. Frequency $f_{\Delta}$ required to test the standard cosmology at radiation temperature $T_{\Delta}$ for a range of values of $G \mu$ with $\alpha=0.1$. The shaded regions indicate where the signal could be detected by the corresponding planned future GW detector.

The power of current and future GW detectors to look back in time using GWs from cosmic strings comes down to their sensitivity to $f_{\Delta}$ for given values of $\alpha$ and $\Gamma G \mu$. Measuring an approximately flat frequency spectrum out to $f_{\Delta}$ would provide strong evidence for radiation domination up to the corresponding temperature $T_{\Delta}$. Thus, $f_{\Delta}$ can be reinterpreted as the frequency needed to test standard cosmology up to temperature $T_{\Delta}$. In figure 4 we show $f_{\Delta}$ as a function of $T_{\Delta}$ for a range of values of $G \mu$ with $\alpha=0.1$ and $\Gamma=50$. Also shown in this figure are the expected sensitivity ranges of LISA, BBO, ET, and CE. All four planned GW detectors could potentially probe the standard cosmology much further back in time than BBN, corresponding to temperatures $T_{\Delta}>5 \mathrm{MeV}$. Note that LIGO does not appear in figure 4 (and figure 5 below) because the GW amplitude of the flat radiation-era plateau lies below the projected sensitivity of the observatory for all values of $\Gamma G \mu$ consistent with the pulsar timing bound of EPTA, as can be seen in figure 3 . However, we show in section 3.3 that LIGO could be sensitive to GW signals from cosmic strings with a non-standard early cosmological history.

In figure 5 we show the expected sensitivities of LISA, BBO, ET, and CE in the $T_{\Delta}-G \mu$ plane. This figure illustrates an important complementarity of the four detectors, corresponding to their respective ranges of frequencies. Indeed, the ability to measure the GW signal over a broad frequency range would be essential to establish the characteristic flat spectrum from the radiation era. Together, figures 4 and 5 also show that these planned observatories could probe the standard cosmological history up to temperatures approaching $T \sim 10^{4} \mathrm{GeV}$, well beyond the BBN era. 


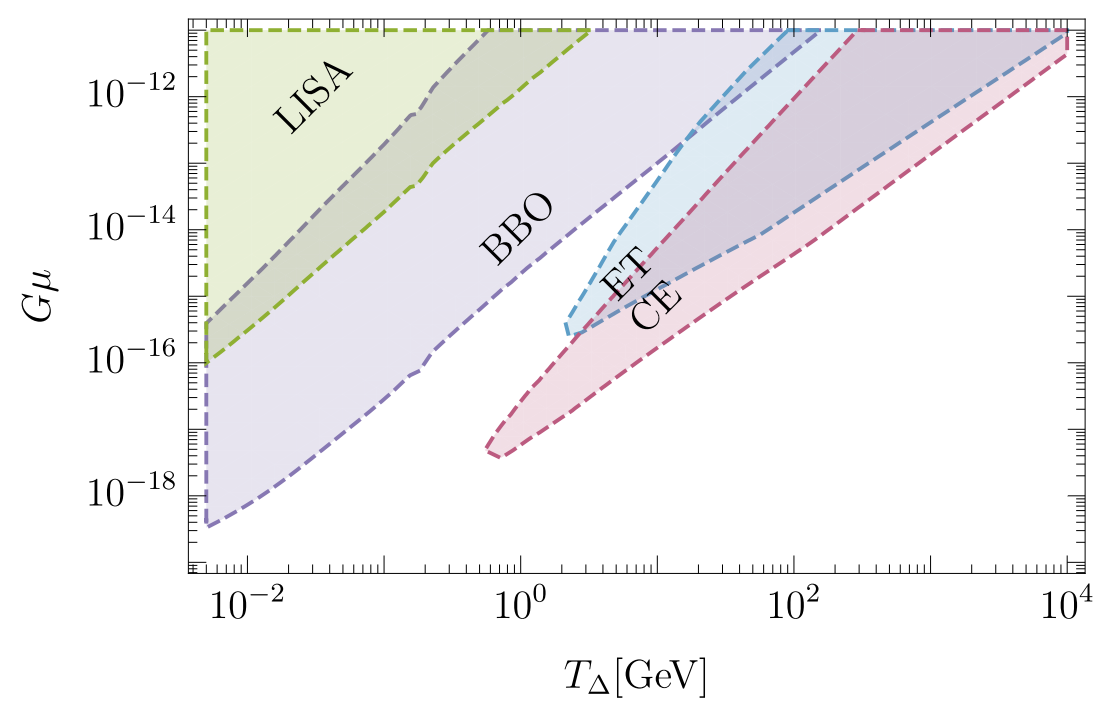

Figure 5. Reach of future GW detectors to test the standard picture of early radiation domination at temperature $T_{\Delta}$ for given values of the string tension $G \mu$. The shaded regions indicate where the signal could be detected by the corresponding planned future GW detector.

\subsection{Probing new degrees of freedom}

Any extension of the SM involving new particles that are thermalized and relativistic in the early universe will contribute to the effective number of energy and entropy degrees of freedom (DOFs), $g_{*}$ and $g_{* S}$. Notably, the minimal supersymmetric extension of the Standard Model predicts $g_{*}=221.5$ (compared to $g_{*}=106.75$ in the SM) at temperatures above the superpartner masses and not counting the gravitino/goldstino. Other approaches to the electroweak hierarchy problem such as the Twin Higgs [110] or NNaturalness [111] also predict many new DOFs near the weak scale. Theories of dark matter with hidden sectors or hidden valleys have also attracted substantial interest in recent years, and frequently give rise to multiple new DOFs, possibly well below the weak scale [112-117]. In some cases, the increase in $g_{*}$ can be enormous [118-120].

New DOFs are already constrained by cosmological observations for masses below a few $\mathrm{MeV}$. Very light states $(m \lesssim \mathrm{eV})$ increase the radiation density at recombination, leaving an imprint on the CMB. This effect is usually expressed as an equivalent number of additional neutrino species [121-125], with measurements limiting $\Delta N_{\text {eff }}^{C M B}<0.30$ [2]. More massive states can avoid the CMB bound, but can modify the expansion rate during $\mathrm{BBN}$, with the limit for masses below $m \lesssim \mathrm{MeV}$ given by $\Delta N_{\text {eff }}^{B B N} \lesssim 0.5$ [126]. In this section we study the effect of new DOFs on the GW spectrum from cosmic strings. We show that detailed GW frequency measurements could probe new, more massive DOFs beyond what can be inferred from the CMB or BBN. Earlier suggestions along this line with a focus on SM degrees of freedom can be found in refs. [127, 128].

To illustrate the generic effect of new massive DOFs on the string GW spectrum without reference to a specific extension of the SM, we model the change by a rapid 
decrease in $g_{*}$ as the temperature falls below the mass threshold $T_{\Delta}$ :

$$
g_{*}(T)=g_{*}^{\mathrm{SM}}(T)+\frac{\Delta g_{*}}{2}\left[1+\tanh \left(10 \frac{T-T_{\Delta}}{T_{\Delta}}\right)\right] \approx\left\{\begin{array}{ll}
g_{*}^{\mathrm{SM}}(T) ; & T<T_{\Delta} \\
g_{*}^{\mathrm{SM}}(T)+\Delta g_{*} ; & T>T_{\Delta}
\end{array} .\right.
$$

An identical modification is assumed for $g_{* S}$, and we use entropy conservation to derive the temperature dependence on the scale factor through the decoupling transition. The resulting dependence of $g_{*}=g_{* S}$ on $T$ is shown in the left panel of figure 6 for $\Delta g_{*}=$ $10^{1}, 10^{2}, 10^{3}$ at $T_{\Delta}=200 \mathrm{GeV}$.

In the right panel of figure 6 we show the effect of changing $g_{*}$ on the GW spectrum from a cosmic string network with $G \mu=10^{-11}$ and $\alpha=0.1$, again for $\Delta g_{*}=10^{1}, 10^{2}, 10^{3}$ at $T_{\Delta}=200 \mathrm{GeV}$. The shaded regions in this panel show the estimated sensitivity bands of SKA, LISA, DECIGO, ET, and CE as in previous figures. A fractional change in $g_{*}$ by order unity or more is seen to produce a significant and potentially observable decrease in the cosmic string GW amplitude above a specific frequency. This transition frequency $f_{\Delta}$ is determined by $T_{\Delta}$ but is independent of $\Delta g_{*}$. For $f \gg f_{\Delta}$, the GW spectrum returns to a flat plateau characteristic of radiation domination $(\mathrm{RD})$ but with a smaller amplitude. The result of figure 6 also shows that future GW detectors could be sensitive to new DOFs with masses relevant to solutions to the electroweak hierarchy problem, possibly even beyond the reach of the LHC. We have checked that this result is insensitive to the precise form of the interpolation function used for $g_{*}$, relative to eq. (3.6), as long as it varies reasonably quickly.

The change in the spectrum shown in the right panel of figure 6 can be understood in terms of the frequency-temperature correspondence derived in section 2.3. As expected, the GW spectrum is only modified above the transition frequency $f_{\Delta}$, which is determined by the temperature (time) at which the standard cosmology is modified. In contrast to this analysis, however, the change in the cosmological evolution from massive decoupling is more subtle than a change in the dilution exponent of the energy density. Even so, a simple analytic estimate of the change in the amplitude is possible.

Since the main contribution to the amplitude at high frequencies is expected to come from deep in the RD era, the Hubble rate and time for large $T$ can be approximated by

$$
H \approx \sqrt{\Delta_{R} \Omega_{R}} H_{0} a^{-2}, \quad t \approx \frac{a^{2}}{2 \sqrt{\Delta_{R} \Omega_{R}}} .
$$

With this simplification, the integral in eq. (2.14) can be written directly in terms of the scale factor to give

$$
\Omega_{\mathrm{GW}}(f) \approx \frac{128 \pi}{9} \Delta_{R} \Omega_{R} \frac{C_{\mathrm{eff}}(n=4)}{\Gamma} \alpha^{1 / 2}(\Gamma G \mu)^{1 / 2},
$$

which agrees well with a similar calculation in ref. [90]. This implies that the amplitude of the RD plateau depends on the number of DOFs via $\Delta_{R}$, and thus

$$
\Omega_{\mathrm{GW}}\left(f \gg f_{\Delta}\right) \approx \Omega_{\mathrm{GW}}^{\mathrm{SM}}(f)\left(\frac{g_{*}^{\mathrm{SM}}}{g_{*}^{\mathrm{SM}}+\Delta g_{*}}\right)^{1 / 3},
$$



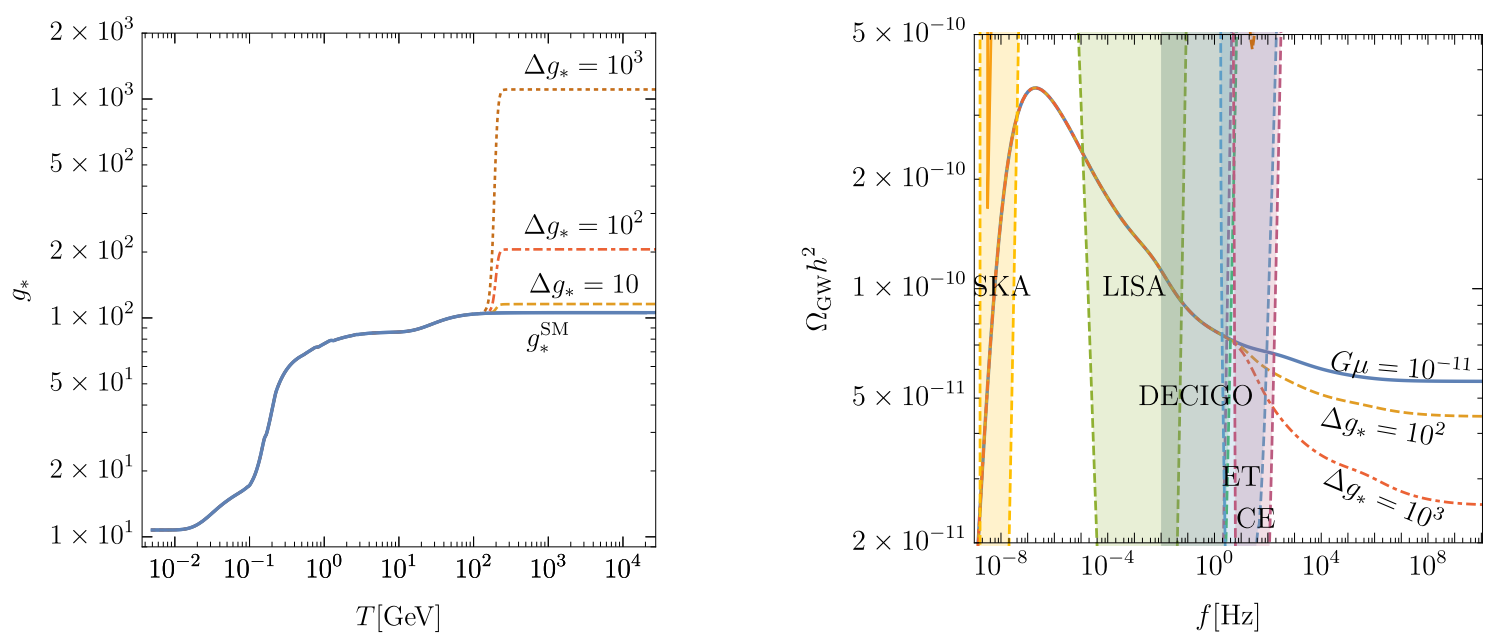

Figure 6. Left panel: illustration of our parametrization of the change in the number of DOFs for $T_{\Delta}=200 \mathrm{GeV}$ and $\Delta g_{*}=10^{1}, 10^{2}, 10^{3}$. Right panel: modification of the $\mathrm{GW}$ spectrum from a cosmic string network with $G \mu=10^{-11}$ and $\alpha=0.1$ for this modification in $g_{*}$. The colored regions in this panel show the expected sensitivities of SKA, LISA, DECIGO, ET, and CE.

where $\Omega_{\mathrm{GW}}^{\mathrm{SM}}$ is the amplitude with only SM DOFs, and we have assumed $g_{*}=g_{* S}$ at high $T$. Therefore an increase of number of DOFs at $T_{\Delta}$ leads to a drop in the amplitude at frequencies above $f_{\Delta}$. In fact, similar changes in the GW amplitude from the RD era from changes in the number of effective SM degrees of freedom at the QCD phase transition and electron-positron decoupling are visible in figures 2 and 3. We also find that the magnitude of the amplitude decrease in eq. (3.9) agrees well with the full numerical result shown in the right panel of figure 6 .

\subsection{Probing new phases of cosmological evolution}

The second type of cosmological modification we consider is an early period in which the expansion of the universe is driven by a new source of energy density prior to the most recent radiation era, leading to a non-standard equation of state in the early universe. For example, an early epoch of matter domination with $\rho \propto a^{-3}$ can arise from a large density of a long-lived massive particle or oscillations of a scalar moduli field in a quadratic potential [12]. Such a period of matter domination ends when the long-lived species decays to the SM. A more exotic class of deviations can arise from the energy density of a scalar field $\phi$ oscillating in a potential of the form $V(\phi) \propto \phi^{N}$, which gives $n=6 N /(N+2)$. In the extreme limit of $N \rightarrow \infty$ we have $n \rightarrow 6$, corresponding to the oscillation energy being dominated by the kinetic energy of the scalar. This behavior arises in models of inflation, quintessence, dark energy, and axions, and is called kination [13, 14, 23]. For all these cases, the universe must settle to radiation domination by the time the temperature reaches $T_{\Delta} \sim 5 \mathrm{MeV}$ in order to preserve the successful predictions of BBN [5]. 
To model the effect of a new cosmological energy source, we parametrize the evolution of the energy density of the universe according to

$$
\rho(t)= \begin{cases}\rho_{s t}\left(t_{\Delta}\right)\left[\frac{a(t)}{a\left(t_{\Delta}\right)}\right]^{-n} ; & t<t_{\Delta} \\ \rho_{s t}(t) ; & t \geq t_{\Delta}\end{cases}
$$

where $\rho_{s t}(t)$ is the standard energy density given by eq. (3.1). In this context, we define $T_{\Delta}$ as the radiation temperature at time $t_{\Delta}$ when the recent period of radiation domination begins. We also focus on the specific cases of $n=3$ and $n=6$ since these bound the envelope of the set of well-motivated possibilities discussed above.

In figure 7 we show the GW spectra from cosmic strings for $\alpha=0.1$ and $G \mu=$ $2 \times 10^{-11}$ (left) and $G \mu=10^{-14}$ (right), together with the modifications to the spectra for early periods of domination with $n=3$ or $n=6$ at representative transition temperatures $T_{\Delta}$. For $G \mu=2 \times 10^{-11}$ on the left we show $T_{\Delta}=5 \mathrm{GeV}$ and $200 \mathrm{GeV}$, and for $G \mu=10^{-14}$ on the right we take $T_{\Delta}=5 \mathrm{MeV}$ and $200 \mathrm{MeV}$. For reference, we also indicate the expected sensitivities of current and future GW detectors.

The onset and shape of the modifications to the GW spectra can be understood in terms of our previous analytical estimates. In particular, eq. (3.5) gives a good approximation of the frequency $f_{\Delta}$ above which the spectrum deviates significantly from the standard cosmology, while eq. (2.21) describes the frequency dependence beyond this. Applying eq. (2.21), we find $\Omega_{G W}\left(f>f_{\Delta}\right) \propto f^{+1}\left(f^{-1}\right)$ for $n=6(3)$. The modifications to the spectrum from the flat plateau of the standard early RD era are drastic and observable provided they occur at low enough frequency to fall within the sensitivity range of current or future experiments.

Relative to the standard cosmology, we also note that an early phase with $n>4$ tends to be easier to observe because it implies a rising amplitude at high frequency. Correspondingly, the experimental sensitivities indicated in figures 4 and 5 are lower bounds on what can be tested for modified cosmologies with $n>4$. Moreover, the left panel of figure 7 shows that future phases of LIGO could probe an $n=6$ modified cosmology up to the transition temperature $T_{\Delta}=200 \mathrm{GeV}$ for $G \mu=2 \times 10^{-11}$, which is about as large a $G \mu$ as possible given current limits from pulsar timing. This range could be extended even further by the proposed ET and CT observatories. However, let us also mention that the maximal GW amplitude is constrained by the total radiation density in GWs, corresponding to $[129,130]$

$$
\int d(\ln f) \Omega_{G W} \lesssim 3.8 \times 10^{-6}
$$

This bound limits the duration of an early phase with $n>4$.

\section{Detection challenges and ways to overcome them}

Up to now we have only studied whether the GW signals from a cosmic string network lie within the sensitivity reach of current and future detectors. In this section we confront the practical challenges of subtracting astrophysical backgrounds and identifying whether such 

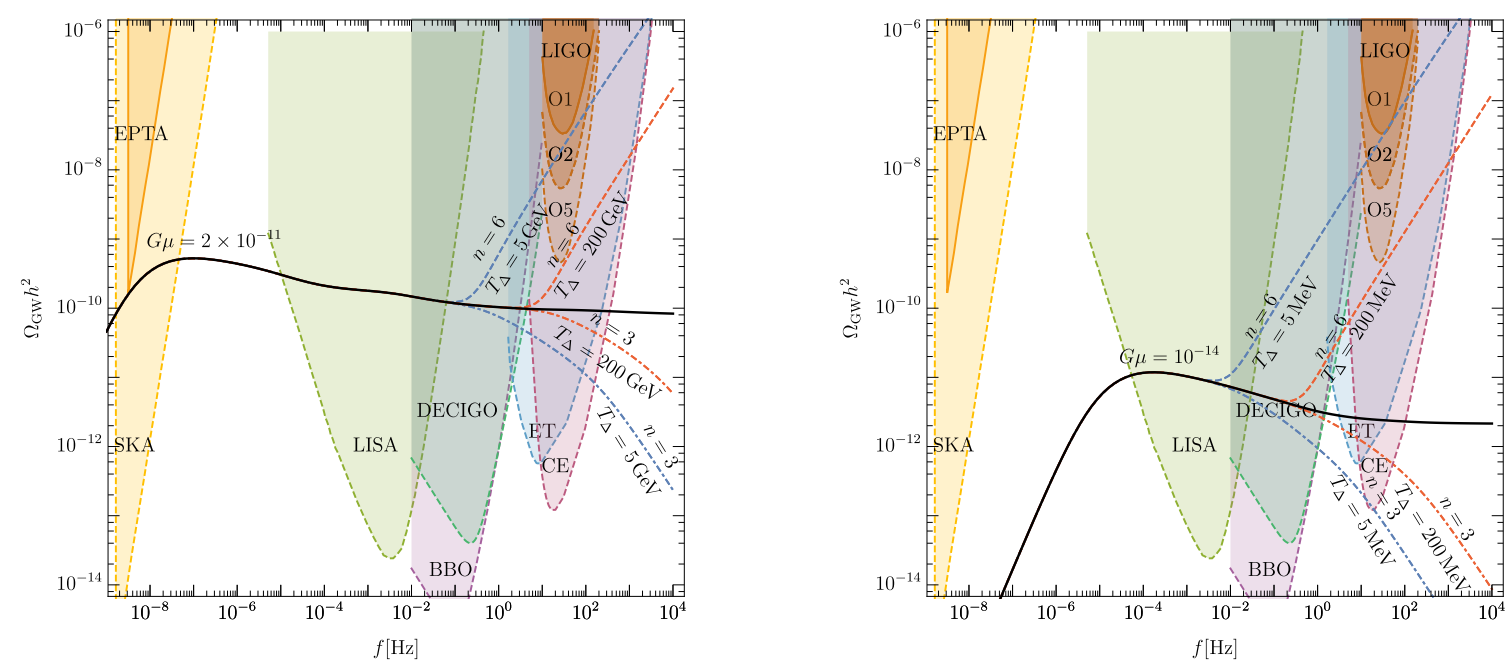

Figure 7. Frequency spectra of gravitational waves $\Omega_{\mathrm{GW}} h^{2}$ sourced by a scaling cosmic string networks with $G \mu=2 \times 10^{-11}$ (left) and $10^{-14}$ (right) with $\alpha=10^{-1}$. The solid black lines show the spectra for a standard cosmological evolution, while the dashed (dash-dotted) lines indicate the result with in an early period of $n=6$ kination ( $n=3$ matter) domination lasting until the temperatures $T_{\Delta}$ listed in the figure. Also shown are the sensitivity bands of current and future GW detectors.

a signal is due to cosmic strings or some other phenomenon. We also examine other potential sources of GW signals and the extent to which they can be distinguished from cosmic strings evolving in a standard or non-standard cosmological background. Finally, we comment on how smaller values of the initial loop size parameter $\alpha$ would impact our results.

\subsection{Astrophysical backgrounds}

The LIGO/Virgo experiment has already observed a number of binary mergers of black holes and neutron stars [100,131-133]. Based on the number of mergers seen and assuming the redshift dependence of the merger rate follows that of star formation, it is anticipated that Advanced LIGO/Virgo will soon begin to detect a stochastic GW background from a collection of weaker, unresolved binary mergers [134, 135]. This background is expected to begin and peak near $f \sim 1000 \mathrm{~Hz}$ with $\Omega_{\mathrm{GW}} \sim 10^{-9}$, and fall off in amplitude as $f^{2 / 3}$ at lower frequencies [136, 137], also putting it within the detection range of space-based detectors such as LISA. The signal from these unresolved mergers will also overlap with, and sometimes overwhelm, the prediction for cosmic strings.

For larger values of $G \mu \gtrsim 10^{-15}$, the lower frequency portion of the cosmic string GW signal could be observed in space-based detectors well above the expected background from binary mergers. This is likely to include a part of the characteristic flat portion of the spectrum from GW emission in the radiation era. In contrast, the higher frequency portion of the cosmic string signal from a standard cosmological history in the sensitivity range of LIGO is likely to be swamped by the binary background. However, let us point out that an enhanced cosmic string signal due to non-standard cosmology with an early 
phase of $n>4$ evolution could potentially rise above background. We also note that the $f^{2 / 3}$ rise in the binary background spectrum has the same frequency scaling as the cosmic string spectrum with an early period of $n=5$ evolution.

Significant effort has been put into finding ways to subtract the background from binary mergers $[29,33,138-144]$. Since the stochastic background from binary mergers comes from those that are not resolved, a promising strategy is to use the improved angular sensitivity of future detectors to identify a great number of them, thereby reducing the portion that contribute to the effective stochastic signal $[29,139,140]$. In particular, ref. [139] suggests that these backgrounds can be removed to the level of $\Omega_{\mathrm{GW}} \sim 10^{-13}$ in future ground-based detector arrays such at ET and CE, while [33, 138] find even better sensitivity for BBO after background subtraction. For LIGO, a statistically optimal search strategy has been proposed recently for identifying unresolved binaries that offers a significant improvement relative to using the traditional cross-correlation method [144]. These studies suggest that the cosmic string GW signals discussed in this work can be separated over background to an extent that they remain a powerful tool to probe the early universe.

\subsection{Distinguishing cosmic strings from other new phenomena}

Cosmic strings are just one of many forms of new physics that can give rise to stochastic GW signals $[29,145,146]$. Other possibilities include primordial inflation [41-43], preheating [147-150], first-order phase transitions [151-153], and other types of topological defects $[154,155]$. Should a new (non-astrophysical) GW signal be observed, identifying the nature of its source will be of paramount importance. Furthermore, if a signal due to cosmic strings is to be used to test the cosmological history of the universe, it must be distinguished from other types of new physics.

A characteristic feature of the GW frequency spectrum from a scaling cosmic string network is the flat plateau at higher frequencies. This feature is difficult to reproduce by most other new sources of GWs. For example, the GW signals from strongly first-order cosmological phase transitions have been studied extensively [156-158], often in connection with electroweak symmetry breaking or baryogenesis [159-165]. The resulting spectrum typically increases following a power law in frequency with a positive exponent up to a peak, and then falls as a power law with a negative exponent at higher frequencies. Other new sources of GWs typically also display such power-law frequency dependence $[29,145,146]$. The partially flat spectrum from a cosmic string network (with standard cosmology) can be distinguished from such a rising and falling spectrum provided the signal can be measured over a reasonably broad frequency range. We emphasize that multiple detectors may be needed to do so. Separating the cosmic string spectrum with an early phase of $n<4$ from the spectrum due to a phase transition would be more challenging, especially if the transition temperature is relatively low. However, even such more complicated scenarios could potentially be identified through precise measurements of the frequency dependence.

A notable exception to the typical split power-law spectrum of new GW sources is the GW signal created by minimal models of inflation. If the inflationary power spectrum is nearly scale invariant, the GW background is expected to be flat over many decades in frequency corresponding to frequencies that reenter the horizon during 
radiation domination $[27,44]$. The stochastic spectrum rises as $f^{-2}$ at lower frequencies $\left(f \lesssim 10^{-16} \mathrm{~Hz}\right)$ correspondng to modes that entered the horizon in the matter era [27, 44]. This is analagous to the flat GW spectrum from cosmic strings during radiation and the rise as $f^{-1 / 2}$ related to the matter era. However, the amplitude inflationary GW spectrum is severely constrained by CMB isotropy and polarization measurements $\left(f \sim 10^{-17} \mathrm{~Hz}\right)$ which constrain the amplitude of the flat part of the spectrum to be less than about $\Omega_{\mathrm{GW}} \lesssim 10^{-15}[54,55]$. A partially flat spectrum due to cosmic strings would then be identifiable simply through its larger amplitude, and possibly by its different power law dependence at lower frequencies. Note that non-minimal models of inflation [166], reheating effects [167], or non-standard neutrino interactions [168] could potentially create a larger signal that rises with frequency. An early phase of kination may also increase the signal amplitude at higher frequencies [48-51].

\subsection{Sensitivity to the loop size parameter $\alpha$}

Recent simulations of cosmic string networks find a population of large loops with initial loop size parameter peaked near $\alpha \simeq 0.1$ [90,91]. We have used this as a fiducial value throughout the work. However, there is some uncertainty in the peak value as well as the distribution around it. Since the amplitude and frequency dependence of the cosmic string GW spectrum depend on $\alpha$, this represents a further challenge to identifying the nature of the early universe through the spectrum.

In figure 8 we show the cosmic string GW spectrum for $G \mu=2 \times 10^{-11}$ while varying the loop size parameter over the range $\alpha=10^{-3}-10^{-1}$. The solid blue line shows the spectrum for the standard cosmological history with $\alpha=0.1$ while the blue band around it shows the effect of reducing this parameter to $\alpha=10^{-2}$ (dark blue band) and $\alpha=10^{-3}$ (light blue band). Similarly, the red dashed (orange dash-dotted) lines indicate the result for $\alpha=0.1$ with in an early period of $n=6$ kination ( $n=3$ matter) domination down to temperature $T_{\Delta}=5 \mathrm{GeV}$. Again, the shaded bands show the effects of reducing $\alpha$ down to $10^{-2}$ and $10^{-3}$.

The dependence on $\alpha$ for the standard cosmological history shown in figure 8 matches our previous analysis in section 3 , with the amplitude of the radiation-era plateau varying as $\Omega \propto(\alpha \Gamma G \mu)^{1 / 2}$ (eq. (3.8)). Furthermore, the frequency at which the spectrum is first modified by non-standard cosmology varies as $f_{\Delta} \propto(\alpha \Gamma G \mu)^{-1 / 2}$ (eq. (3.5)). For early matter domination $(n=3)$ the modifications to the frequency and amplitude cancel out, leaving a falling slope at high frequency unchanged. In contrast, for early kination $(n=6)$ the changes to the frequency and amplitude add to give a linear relation $\Omega_{G W} \propto \alpha^{1}$. While an uncertainty in $\alpha$ would complicate the identification of a transition temperature $T_{\Delta}$, it does not make it impossible. In principle, the combination $\alpha \Gamma G \mu$ could be extracted from the amplitude of a flat radiation plateau and then applied to obtain $T_{\Delta}$ from an observation of $f_{\Delta}$ via eq. (3.5). 


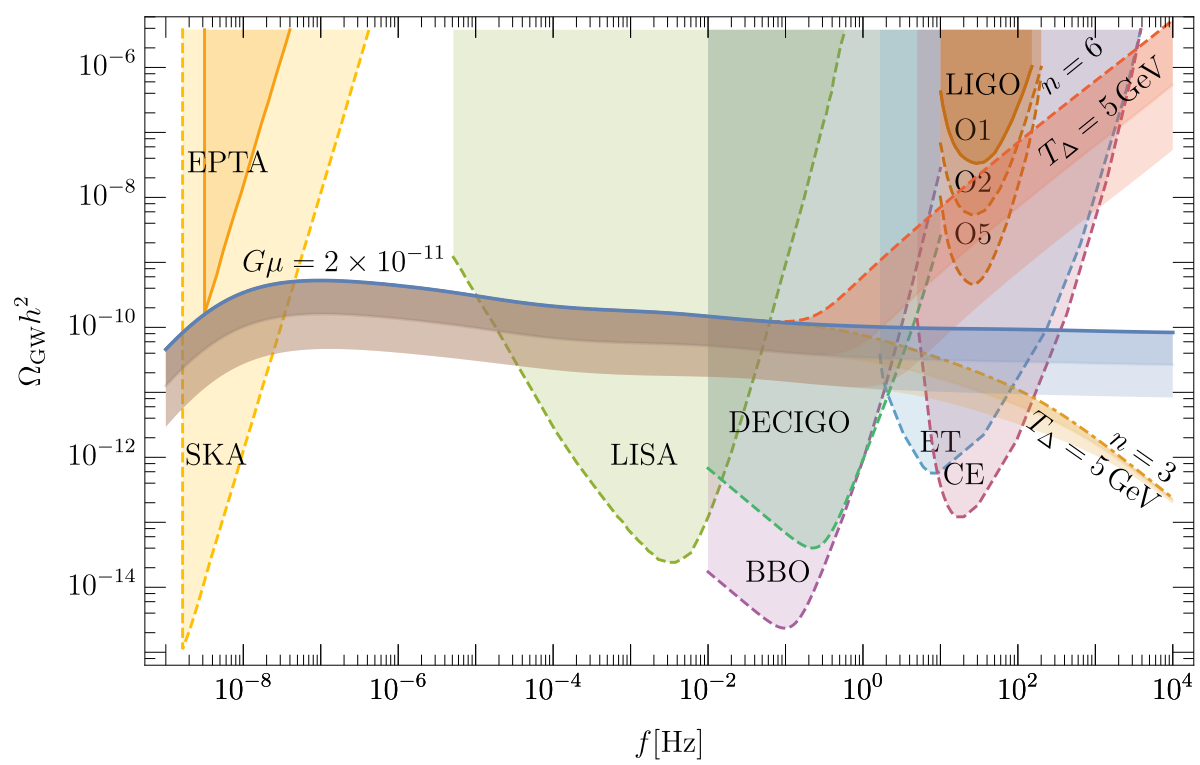

Figure 8. Gravitational wave spectra from a cosmic string network with $G \mu=2 \times 10^{-11}$ and $\alpha=10^{-3}-10^{-1}$. The solid blue line shows the spectrum for the standard cosmological history with $\alpha=0.1$ while the dark (light) blue band around it shows the effect of reducing to $\alpha=10^{-2}\left(10^{-3}\right)$. The red dashed (orange dash-dotted) lines indicate the result for $\alpha=0.1$ with in an early period of $n=6$ kination ( $n=3$ matter) domination lasting down to temperature $T_{\Delta}=5 \mathrm{MeV}$. As before, the dark (light) colored bands around the lines show the effects of reducing the initial loop size to $\alpha=10^{-2}\left(10^{-3}\right)$.

\section{Conclusions}

Standard cosmology maintains that an era of radiation domination began in the early universe and was followed by matter domination, which then ultimately yields to an increasing acceleration era dominated by the cosmological constant. This framework is well tested and is found to be self-consistent by a multitude of experimental probes including measurements of the CMB, supernovae, large-scale structure, and abundances of nuclei as predicted by BBN epoch.

Unfortunately, the traditional experimental probes reach back only as far as BBN, which corresponds to temperatures below only about $5 \mathrm{MeV}$. There are many ideas for new physics above $5 \mathrm{MeV}$ that disrupt the standard cosmology, whether it be through a different scaling phase other than radiation domination (e.g., matter or kination domination), or through extra degrees of freedom beyond the known Standard Model ones that substantially modify radiation era dynamics. Therefore, testing for new physics, and an altered cosmological evolution at temperatures greater than $5 \mathrm{MeV}$, requires new methods. The potential answer is gravitational waves, whose very early origins pass safely through recombination and BBN, which scrambles the otherwise powerful CMB probes and BBN constraints.

A strong early universe source of GWs must be present in order to probe the effects that cosmological evolution can have on it. Furthermore, this source must have a reasonably 
well understood emission spectrum - analogous to the standard candles of supernovae with which to propagate through various assumed cosmological histories and compare with observational data. A prime candidate for this is cosmic strings, whose network formation and emission spectrum has been well studied and understood, particularly featuring a long flat plateau at high frequency during standard radiation dominated era. Another reason cosmic stings are useful GW sources to consider is that they are generically expected in a wide variety of high-scale theories of particle physics, ranging from unified field theories containing abelian factors to fundamental string theory.

We have assumed the existence of cosmic strings in the early universe and have worked out the GW relic abundance vs. frequency spectrum for many different cosmic string tensions $G \mu$. We have reiterated previous results in the literature that GWs are an excellent way to constrain and find evidence for cosmic strings even within standard cosmological evolution (see figure 2). In addition, and what is central to our study, the GWs from cosmic strings enable the probing of modifications of early universe cosmology in regimes that no other probe can.

We studied two main ways that early universe cosmology can change. First, we studied the effect of having a very large number of additional degrees of freedom present in the spectrum at high energy. If the degrees of freedom are present down to temperature $T_{\Delta}$ one finds that there is a frequency $f_{\Delta}$ above which the GW energy density is altered compared to the expectations of standard cosmology (with SM degrees of freedom). The signal for the onset of a high number of degrees of freedom is therefore standard $\Omega_{\mathrm{GW}}(f)$ vs. $f$ for cosmic strings up to $f_{\Delta}$ and then a fall-off for $f>f_{\Delta}$ compared to expectations. Figure 6 shows the effect in the $\Omega_{\mathrm{GW}}(f)$ vs. $f$ plane.

A second example is GWs from cosmic strings evolving in a non-standard phase, either of an early matter domination phase $(n=3)$ or an early kination $(n=6)$ phase. The early matter phase may be due the presence of a large density of heavy new physics states that later decay bringing the universe back to radiation era, which is needed to satisfy BBN constraints. In other words, the universe transitions from radiation domination at very high temperatures to matter domination (at $T$ comparable to mass of long-lived heavy new particles) and then back to radiation domination (by decay of said particles) before the onset of BBN. The kination $(n=6)$ phase arises from oscillating scalar moduli in the early universe, which then decay. This leads to a cosmological history of very early radiation domination to kination domination (oscillation energy dominating) and back to radiation (by decay of the moduli).

The ability to probe these alternative cosmological histories well by cosmic strings partly derives from the property that cosmic strings rapidly enter a scaling regime, which means their energy density scales with scale factor $a$ exactly the same as the dominant energy density of the universe. If there is an early matter domination phase then GW energy density scales like $a^{3}$ during that phase, and if there is an early kination phase, cosmic strings will scale like $a^{6}$ during that phase. The scaling behavior of cosmic strings means that the energy density of the GWs emitted will be altered substantially through its non-standard redshifting. Our numerical work shows the effect quantitatively, which leads to a sharp fall-off in $\Omega_{\mathrm{GW}}(f)$ at high frequency $f$ (corresponding to the new phase era) if 
there is early matter domination, and a sharp rise in $\Omega_{\mathrm{GW}}(f)$ if there is an early kination phase. The results are illustrated in figure 7 .

GW detectors have given us a window to early universe cosmology complementary to any other probes previously developed. We have argued that a strong and well-understood source of GWs in the early universe could give us unprecedented ability to probe cosmological energy evolution of the early universe far earlier than previously attainable. We have also demonstrated that cosmic strings, if they exist, would be excellent standard candles to achieve these aims.

\section{Acknowledgments}

We thank Jose J. Blanco-Pillado, Nikita Blinov, Bhaskar Dutta, Vuk Mandic, and David McKeen for helpful discussions. ML and YC would like to thank the Mainz Institute for Theoretical Physics (MITP) for its hospitality and support. YC is supported in part by the US Department of Energy (DOE) grant DE-SC0008541. ML is supported by the United Kingdom STFC Grant ST/P000258/1 and by the Polish MNiSW grant IP2015 043174. DEM is supported by a Discovery Grant from the Natural Sciences and Engineering Research Council of Canada (NSERC), and TRIUMF, which receives federal funding via a contribution agreement with the National Research Council of Canada (NRC). JDW is supported in part by the DOE under grant DE-SC0007859.

Open Access. This article is distributed under the terms of the Creative Commons Attribution License (CC-BY 4.0), which permits any use, distribution and reproduction in any medium, provided the original author(s) and source are credited.

\section{References}

[1] R. Hill, K.W. Masui and D. Scott, The spectrum of the universe, The Universe 72 (2018) 663 [arXiv: 1802.03694] [INSPIRE].

[2] Planck collaboration, Planck 2018 results. VI. Cosmological parameters, arXiv: 1807.06209 [INSPIRE].

[3] M. Kawasaki, K. Kohri and N. Sugiyama, Cosmological constraints on late time entropy production, Phys. Rev. Lett. 82 (1999) 4168 [astro-ph/9811437] [INSPIRE].

[4] M. Kawasaki, K. Kohri and N. Sugiyama, MeV scale reheating temperature and thermalization of neutrino background, Phys. Rev. D 62 (2000) 023506 [astro-ph/0002127] [INSPIRE].

[5] S. Hannestad, What is the lowest possible reheating temperature?, Phys. Rev. D 70 (2004) 043506 [astro-ph/0403291] [INSPIRE].

[6] A.H. Guth, The inflationary universe: a possible solution to the horizon and flatness problems, Phys. Rev. D 23 (1981) 347 [InSPIRE].

[7] A.D. Linde, A new inflationary universe scenario: a possible solution of the horizon, flatness, homogeneity, isotropy and primordial monopole problems, Phys. Lett. B 108 (1982) 389 [Adv. Ser. Astrophys. Cosmol. 3 (1987) 149] [INSPIRE]. 
[8] A. Albrecht and P.J. Steinhardt, Cosmology for grand unified theories with radiatively induced symmetry breaking, Phys. Rev. Lett. 48 (1982) 1220 [INSPIRE].

[9] Planck collaboration, Planck 2018 results. X. Constraints on inflation, arXiv:1807.06211 [INSPIRE].

[10] L.A. Boyle and A. Buonanno, Relating gravitational wave constraints from primordial nucleosynthesis, pulsar timing, laser interferometers and the CMB: implications for the early Universe, Phys. Rev. D 78 (2008) 043531 [arXiv: 0708.2279] [INSPIRE].

[11] E.W. Kolb and M.S. Turner, The early universe, Front. Phys. 69 (1990) 1 [InSPIRE].

[12] T. Moroi and L. Randall, Wino cold dark matter from anomaly mediated SUSY breaking, Nucl. Phys. B 570 (2000) 455 [hep-ph/9906527] [INSPIRE].

[13] P. Salati, Quintessence and the relic density of neutralinos, Phys. Lett. B 571 (2003) 121 [astro-ph/0207396] [INSPIRE].

[14] D.J.H. Chung, L.L. Everett and K.T. Matchev, Inflationary cosmology connecting dark energy and dark matter, Phys. Rev. D 76 (2007) 103530 [arXiv:0704.3285] [INSPIRE].

[15] G.B. Gelmini and P. Gondolo, Ultra-cold WIMPs: relics of non-standard pre-BBN cosmologies, JCAP 10 (2008) 002 [arXiv:0803.2349] [INSPIRE].

[16] L. Visinelli and P. Gondolo, Axion cold dark matter in non-standard cosmologies, Phys. Rev. D 81 (2010) 063508 [arXiv:0912.0015] [InSPIRE].

[17] A.L. Erickcek, The dark matter annihilation boost from low-temperature reheating, Phys. Rev. D 92 (2015) 103505 [arXiv: 1504.03335] [INSPIRE].

[18] B. Dutta, E. Jimenez and I. Zavala, Dark matter relics and the expansion rate in scalar-tensor theories, JCAP 06 (2017) 032 [arXiv: 1612.05553] [INSPIRE].

[19] J.T. Giblin, G. Kane, E. Nesbit, S. Watson and Y. Zhao, Was the universe actually radiation dominated prior to nucleosynthesis?, Phys. Rev. D 96 (2017) 043525 [arXiv: 1706. 08536] [INSPIRE].

[20] L. Visinelli, (Non-)thermal production of WIMPs during kination, Symmetry 10 (2018) 546 [arXiv:1710.11006] [INSPIRE].

[21] L. Visinelli, Light axion-like dark matter must be present during inflation, Phys. Rev. D 96 (2017) 023013 [arXiv: 1703.08798] [INSPIRE].

[22] L. Visinelli and J. Redondo, Axion miniclusters in modified cosmological histories, arXiv: 1808.01879 [INSPIRE].

[23] V. Poulin, T.L. Smith, D. Grin, T. Karwal and M. Kamionkowski, Cosmological implications of ultralight axionlike fields, Phys. Rev. D 98 (2018) 083525 [arXiv: 1806. 10608] [INSPIRE].

[24] B. Dutta, C.S. Fong, E. Jimenez and E. Nardi, A cosmological pathway to testable leptogenesis, JCAP 10 (2018) 025 [arXiv: 1804.07676] [INSPIRE].

[25] K. Redmond, A. Trezza and A.L. Erickcek, Growth of dark matter perturbations during kination, Phys. Rev. D 98 (2018) 063504 [arXiv:1807.01327] [INSPIRE].

[26] A.E. Nelson and H. Xiao, Axion cosmology with early matter domination, Phys. Rev. D 98 (2018) 063516 [arXiv: 1807.07176] [inSPIRE]. 
[27] B. Allen, The stochastic gravity wave background: sources and detection, in Relativistic gravitation and gravitational radiation. Proceedings, School of Physics, Les Houches, France, 26 September-6 October 1995, pg. 373 [gr-qc/9604033] [INSPIRE].

[28] Y. Cui, M. Lewicki, D.E. Morrissey and J.D. Wells, Cosmic archaeology with gravitational waves from cosmic strings, Phys. Rev. D 97 (2018) 123505 [arXiv:1711.03104] [InSPIRE].

[29] C. Caprini and D.G. Figueroa, Cosmological backgrounds of gravitational waves, Class. Quant. Grav. 35 (2018) 163001 [arXiv:1801.04268] [INSPIRE].

[30] Ligo Scientific, Virgo, 1M2H, Dark Energy Camera GW-E, Des, DLT40, Las Cumbres Observatory, VINROUGE and MASTER collaborations, $A$ gravitational-wave standard siren measurement of the Hubble constant, Nature $\mathbf{5 5 1}$ (2017) 85 [arXiv: 1710.05835] [INSPIRE].

[31] LIGO SCIENTIFIC and VIRGO collaborations, Characterization of the LIGO detectors during their sixth science run, Class. Quant. Grav. 32 (2015) 115012 [arXiv:1410.7764] [INSPIRE].

[32] LISA collaboration, Laser Interferometer Space Antenna, arXiv:1702.00786 [INSPIRE].

[33] K. Yagi and N. Seto, Detector configuration of DECIGO/BBO and identification of cosmological neutron-star binaries, Phys. Rev. D 83 (2011) 044011 [Erratum ibid. D 95 (2017) 109901] [arXiv: 1101.3940] [INSPIRE].

[34] M. Punturo et al., The Einstein telescope: a third-generation gravitational wave observatory, Class. Quant. Grav. 27 (2010) 194002 [INSPIRE].

[35] S. Hild et al., Sensitivity studies for third-generation gravitational wave observatories, Class. Quant. Grav. 28 (2011) 094013 [arXiv: 1012.0908] [INSPIRE].

[36] LIGO SCIENTIFIC collaboration, Exploring the sensitivity of next generation gravitational wave detectors, Class. Quant. Grav. 34 (2017) 044001 [arXiv: 1607.08697] [InSPIRE].

[37] A. Vilenkin, Cosmic strings and domain walls, Phys. Rept. 121 (1985) 263 [INSPIRE].

[38] R.R. Caldwell and B. Allen, Cosmological constraints on cosmic string gravitational radiation, Phys. Rev. D 45 (1992) 3447 [INSPIRE].

[39] M.B. Hindmarsh and T.W.B. Kibble, Cosmic strings, Rept. Prog. Phys. 58 (1995) 477 [hep-ph/9411342] [INSPIRE].

[40] A. Vilenkin and E.P.S. Shellard, Cosmic strings and other topological defects, Cambridge University Press, Cambridge, U.K. (2000) [INSPIRE].

[41] L.P. Grishchuk, Amplification of gravitational waves in an istropic universe, Sov. Phys. JETP 40 (1975) 409 [Zh. Eksp. Teor. Fiz. 67 (1974) 825] [INSPIRE].

[42] A.A. Starobinsky, Spectrum of relict gravitational radiation and the early state of the universe, JETP Lett. 30 (1979) 682 [Pisma Zh. Eksp. Teor. Fiz. 30 (1979) 719] [inSPIRE].

[43] B. Allen, The stochastic gravity wave background in inflationary universe models, Phys. Rev. D 37 (1988) 2078 [INSPIRE].

[44] M.S. Turner, M.J. White and J.E. Lidsey, Tensor perturbations in inflationary models as a probe of cosmology, Phys. Rev. D 48 (1993) 4613 [astro-ph/9306029] [INSPIRE].

[45] N. Seto and J. Yokoyama, Probing the equation of state of the early universe with a space laser interferometer, J. Phys. Soc. Jap. 72 (2003) 3082 [gr-qc/0305096] [InSPIRE]. 
[46] K. Nakayama, S. Saito, Y. Suwa and J. Yokoyama, Probing reheating temperature of the universe with gravitational wave background, JCAP 06 (2008) 020 [arXiv:0804.1827] [INSPIRE].

[47] K. Nakayama, S. Saito, Y. Suwa and J. Yokoyama, Space laser interferometers can determine the thermal history of the early universe, Phys. Rev. D 77 (2008) 124001 [arXiv:0802.2452] [INSPIRE].

[48] M. Giovannini, Gravitational waves constraints on postinflationary phases stiffer than radiation, Phys. Rev. D 58 (1998) 083504 [hep-ph/9806329] [INSPIRE].

[49] A. Riazuelo and J.-P. Uzan, Quintessence and gravitational waves, Phys. Rev. D 62 (2000) 083506 [astro-ph/0004156] [INSPIRE].

[50] V. Sahni, M. Sami and T. Souradeep, Relic gravity waves from brane world inflation, Phys. Rev. D 65 (2002) 023518 [gr-qc/0105121] [INSPIRE].

[51] H. Tashiro, T. Chiba and M. Sasaki, Reheating after quintessential inflation and gravitational waves, Class. Quant. Grav. 21 (2004) 1761 [gr-qc/0307068] [INSPIRE].

[52] M.C. Guzzetti, N. Bartolo, M. Liguori and S. Matarrese, Gravitational waves from inflation, Riv. Nuovo Cim. 39 (2016) 399 [arXiv: 1605.01615] [INSPIRE].

[53] K.N. Ananda, C. Clarkson and D. Wands, The cosmological gravitational wave background from primordial density perturbations, Phys. Rev. D 75 (2007) 123518 [gr-qc/0612013] [INSPIRE].

[54] T.L. Smith, M. Kamionkowski and A. Cooray, Direct detection of the inflationary gravitational wave background, Phys. Rev. D 73 (2006) 023504 [astro-ph/0506422] [INSPIRE].

[55] P.D. Lasky et al., Gravitational-wave cosmology across 29 decades in frequency, Phys. Rev. X 6 (2016) 011035 [arXiv:1511.05994] [INSPIRE].

[56] H.B. Nielsen and P. Olesen, Vortex line models for dual strings, Nucl. Phys. B 61 (1973) 45 [INSPIRE].

[57] T.W.B. Kibble, Topology of cosmic domains and strings, J. Phys. A 9 (1976) 1387 [INSPIRE].

[58] E.J. Copeland, R.C. Myers and J. Polchinski, Cosmic F and D strings, JHEP 06 (2004) 013 [hep-th/0312067] [INSPIRE].

[59] G. Dvali and A. Vilenkin, Formation and evolution of cosmic D strings, JCAP 03 (2004) 010 [hep-th/0312007] [INSPIRE].

[60] J. Polchinski, Introduction to cosmic F- and D-strings, in String theory: from gauge interactions to cosmology. Proceedings, NATO Advanced Study Institute, Cargese, France, 7-19 June 2004, pg. 229 [hep-th/0412244] [INSPIRE].

[61] M.G. Jackson, N.T. Jones and J. Polchinski, Collisions of cosmic F and D-strings, JHEP 10 (2005) 013 [hep-th/0405229] [INSPIRE].

[62] S.-H. Henry Tye, I. Wasserman and M. Wyman, Scaling of multi-tension cosmic superstring networks, Phys. Rev. D 71 (2005) 103508 [Erratum ibid. D 71 (2005) 129906] [astro-ph/0503506] [INSPIRE].

[63] T. Charnock, A. Avgoustidis, E.J. Copeland and A. Moss, CMB constraints on cosmic strings and superstrings, Phys. Rev. D 93 (2016) 123503 [arXiv:1603.01275] [INSPIRE]. 
[64] A. Vilenkin, Gravitational radiation from cosmic strings, Phys. Lett. B 107 (1981) 47 [INSPIRE].

[65] T. Vachaspati and A. Vilenkin, Gravitational radiation from cosmic strings, Phys. Rev. D 31 (1985) 3052 [INSPIRE].

[66] N. Turok, Grand unified strings and galaxy formation, Nucl. Phys. B 242 (1984) 520 [INSPIRE].

[67] C.J. Burden, Gravitational radiation from a particular class of cosmic strings, Phys. Lett. B 164 (1985) 277 [INSPIRE].

[68] A. Albrecht and N. Turok, Evolution of cosmic strings, Phys. Rev. Lett. 54 (1985) 1868 [INSPIRE].

[69] D.P. Bennett and F.R. Bouchet, Evidence for a scaling solution in cosmic string evolution, Phys. Rev. Lett. 60 (1988) 257 [INSPIRE].

[70] B. Allen and E.P.S. Shellard, Cosmic string evolution: a numerical simulation, Phys. Rev. Lett. 64 (1990) 119 [INSPIRE].

[71] Ya. B. Zeldovich and M. Yu. Khlopov, On the concentration of relic magnetic monopoles in the universe, Phys. Lett. B 79 (1978) 239 [InSPIRE].

[72] J. Preskill, Cosmological production of superheavy magnetic monopoles, Phys. Rev. Lett. 43 (1979) 1365 [INSPIRE].

[73] Ya. B. Zeldovich, I. Yu. Kobzarev and L.B. Okun, Cosmological consequences of the spontaneous breakdown of discrete symmetry, Zh. Eksp. Teor. Fiz. 67 (1974) 3 [Sov. Phys. JETP 40 (1974) 1] [INSPIRE].

[74] K.D. Olum and J.J. Blanco-Pillado, Radiation from cosmic string standing waves, Phys. Rev. Lett. 84 (2000) 4288 [astro-ph/9910354] [INSPIRE].

[75] J.N. Moore, E.P.S. Shellard and C.J. A.P. Martins, On the evolution of Abelian-Higgs string networks, Phys. Rev. D 65 (2002) 023503 [hep-ph/0107171] [InSPIRE].

[76] G. Vincent, N.D. Antunes and M. Hindmarsh, Numerical simulations of string networks in the Abelian Higgs model, Phys. Rev. Lett. 80 (1998) 2277 [hep-ph/9708427] [INSPIRE].

[77] N. Bevis, M. Hindmarsh, M. Kunz and J. Urrestilla, CMB power spectrum contribution from cosmic strings using field-evolution simulations of the Abelian Higgs model, Phys. Rev. D 75 (2007) 065015 [astro-ph/0605018] [INSPIRE].

[78] D.G. Figueroa, M. Hindmarsh and J. Urrestilla, Exact scale-invariant background of gravitational waves from cosmic defects, Phys. Rev. Lett. 110 (2013) 101302 [arXiv: 1212.5458] [INSPIRE].

[79] T. Helfer, J.C. Aurrekoetxea and E.A. Lim, Cosmic string loop collapse in full general relativity, arXiv: 1808.06678 [INSPIRE].

[80] M. Srednicki and S. Theisen, Nongravitational decay of cosmic strings, Phys. Lett. B 189 (1987) 397 [INSPIRE].

[81] A. Vilenkin and T. Vachaspati, Radiation of Goldstone bosons from cosmic strings, Phys. Rev. D 35 (1987) 1138 [INSPIRE].

[82] T. Damour and A. Vilenkin, Cosmic strings and the string dilaton, Phys. Rev. Lett. 78 (1997) 2288 [gr-qc/9610005] [INSPIRE]. 
[83] Y. Cui and D.E. Morrissey, Non-thermal dark matter from cosmic strings, Phys. Rev. D 79 (2009) 083532 [arXiv:0805.1060] [INSPIRE].

[84] A.J. Long, J.M. Hyde and T. Vachaspati, Cosmic strings in hidden sectors: 1. Radiation of Standard Model particles, JCAP 09 (2014) 030 [arXiv:1405.7679] [INSPIRE].

[85] V. Vanchurin, K.D. Olum and A. Vilenkin, Scaling of cosmic string loops, Phys. Rev. D 74 (2006) 063527 [gr-qc/0511159] [INSPIRE].

[86] C.J. A.P. Martins and E.P.S. Shellard, Fractal properties and small-scale structure of cosmic string networks, Phys. Rev. D 73 (2006) 043515 [astro-ph/0511792] [INSPIRE].

[87] K.D. Olum and V. Vanchurin, Cosmic string loops in the expanding universe, Phys. Rev. D 75 (2007) 063521 [astro-ph/0610419] [INSPIRE].

[88] C. Ringeval, M. Sakellariadou and F. Bouchet, Cosmological evolution of cosmic string loops, JCAP 02 (2007) 023 [astro-ph/0511646] [INSPIRE].

[89] J.J. Blanco-Pillado, K.D. Olum and B. Shlaer, Large parallel cosmic string simulations: new results on loop production, Phys. Rev. D 83 (2011) 083514 [arXiv:1101.5173] [INSPIRE].

[90] J.J. Blanco-Pillado and K.D. Olum, Stochastic gravitational wave background from smoothed cosmic string loops, Phys. Rev. D 96 (2017) 104046 [arXiv:1709.02693] [INSPIRE].

[91] J.J. Blanco-Pillado, K.D. Olum and B. Shlaer, The number of cosmic string loops, Phys. Rev. D 89 (2014) 023512 [arXiv:1309.6637] [InSPIRE].

[92] C.J. A.P. Martins and E.P.S. Shellard, String evolution with friction, Phys. Rev. D 53 (1996) 575 [hep-ph/9507335] [INSPIRE].

[93] C.J. A.P. Martins and E.P.S. Shellard, Quantitative string evolution, Phys. Rev. D 54 (1996) 2535 [hep-ph/9602271] [INSPIRE].

[94] C.J. A.P. Martins and E.P.S. Shellard, Extending the velocity dependent one scale string evolution model, Phys. Rev. D 65 (2002) 043514 [hep-ph/0003298] [INSPIRE].

[95] P.P. Avelino and L. Sousa, Scaling laws for weakly interacting cosmic (super)string and p-brane networks, Phys. Rev. D 85 (2012) 083525 [arXiv:1202.6298] [INSPIRE].

[96] L. Sousa and P.P. Avelino, Stochastic gravitational wave background generated by cosmic string networks: velocity-dependent one-scale model versus scale-invariant evolution, Phys. Rev. D 88 (2013) 023516 [arXiv: 1304.2445] [INSPIRE].

[97] J.M. Quashnock and D.N. Spergel, Gravitational selfinteractions of cosmic strings, Phys. Rev. D 42 (1990) 2505 [INSPIRE].

[98] LIGO Scientific collaboration, Advanced LIGO, Class. Quant. Grav. 32 (2015) 074001 [arXiv: 1411.4547] [INSPIRE].

[99] E. Thrane and J.D. Romano, Sensitivity curves for searches for gravitational-wave backgrounds, Phys. Rev. D 88 (2013) 124032 [arXiv:1310.5300] [INSPIRE].

[100] LIGO SCIENTIFIC and VIRGO collaborations, GW150914: implications for the stochastic gravitational wave background from binary black holes, Phys. Rev. Lett. 116 (2016) 131102 [arXiv: 1602.03847] [INSPIRE].

[101] LigO ScIENTIFIC and ViRgo collaborations, Constraints on cosmic strings using data from the first advanced LIGO observing run, Phys. Rev. D 97 (2018) 102002 [arXiv: 1712.01168] [INSPIRE]. 
[102] N. Bartolo et al., Science with the space-based interferometer LISA. IV: probing inflation with gravitational waves, JCAP 12 (2016) 026 [arXiv:1610.06481] [INSPIRE].

[103] R. van Haasteren et al., Placing limits on the stochastic gravitational-wave background using European Pulsar Timing Array data, Mon. Not. Roy. Astron. Soc. 414 (2011) 3117 [Erratum ibid. 425 (2012) 1597] [arXiv:1103.0576] [INSPIRE].

[104] G. Janssen et al., Gravitational wave astronomy with the SKA, PoS (AASKA14) 037 (2015) [arXiv: 1501.00127] [INSPIRE].

[105] J.J. Blanco-Pillado, K.D. Olum and X. Siemens, New limits on cosmic strings from gravitational wave observation, Phys. Lett. B 778 (2018) 392 [arXiv:1709.02434] [INSPIRE].

[106] C. Ringeval and T. Suyama, Stochastic gravitational waves from cosmic string loops in scaling, JCAP 12 (2017) 027 [arXiv: 1709.03845] [INSPIRE].

[107] S. Kuroyanagi, K. Miyamoto, T. Sekiguchi, K. Takahashi and J. Silk, Forecast constraints on cosmic strings from future CMB, pulsar timing and gravitational wave direct detection experiments, Phys. Rev. D 87 (2013) 023522 [Erratum ibid. D 87 (2013) 069903] [arXiv: 1210.2829] [INSPIRE].

[108] Planck collaboration, Planck 2015 results. XIII. Cosmological parameters, Astron. Astrophys. 594 (2016) A13 [arXiv:1502.01589] [INSPIRE].

[109] G. Bélanger, F. Boudjema, A. Pukhov and A. Semenov, MicrOMEGAs4.1: two dark matter candidates, Comput. Phys. Commun. 192 (2015) 322 [arXiv:1407.6129] [INSPIRE].

[110] Z. Chacko, H.-S. Goh and R. Harnik, The twin Higgs: natural electroweak breaking from mirror symmetry, Phys. Rev. Lett. 96 (2006) 231802 [hep-ph/0506256] [INSPIRE].

[111] N. Arkani-Hamed, T. Cohen, R.T. D'Agnolo, A. Hook, H.D. Kim and D. Pinner, Solving the hierarchy problem at reheating with a large number of degrees of freedom, Phys. Rev. Lett. 117 (2016) 251801 [arXiv: 1607.06821] [INSPIRE].

[112] E.W. Kolb, D. Seckel and M.S. Turner, The shadow world, Nature 314 (1985) 415 [INSPIRE].

[113] H.M. Hodges, Mirror baryons as the dark matter, Phys. Rev. D 47 (1993) 456 [InSPIRE].

[114] M.J. Strassler and K.M. Zurek, Echoes of a hidden valley at hadron colliders, Phys. Lett. B 651 (2007) 374 [hep-ph/0604261] [INSPIRE].

[115] J.L. Feng, H. Tu and H.-B. Yu, Thermal relics in hidden sectors, JCAP 10 (2008) 043 [arXiv:0808.2318] [INSPIRE].

[116] R. Foot, Mirror dark matter: cosmology, galaxy structure and direct detection, Int. J. Mod. Phys. A 29 (2014) 1430013 [arXiv: 1401.3965] [InSPIRE].

[117] P. Adshead, Y. Cui and J. Shelton, Chilly dark sectors and asymmetric reheating, JHEP 06 (2016) 016 [arXiv: 1604.02458] [INSPIRE].

[118] D.E. Kaplan and R. Rattazzi, Large field excursions and approximate discrete symmetries from a clockwork axion, Phys. Rev. D 93 (2016) 085007 [arXiv:1511.01827] [INSPIRE].

[119] G.F. Giudice and M. McCullough, A clockwork theory, JHEP 02 (2017) 036 [arXiv: 1610.07962] [INSPIRE].

[120] A. Soni and Y. Zhang, Hidden SU(N) glueball dark matter, Phys. Rev. D 93 (2016) 115025 [arXiv: 1602.00714] [INSPIRE]. 
[121] C. Brust, D.E. Kaplan and M.T. Walters, New light species and the CMB, JHEP 12 (2013) 058 [arXiv: 1303.5379] [INSPIRE].

[122] Z. Chacko, Y. Cui, S. Hong and T. Okui, Hidden dark matter sector, dark radiation and the CMB, Phys. Rev. D 92 (2015) 055033 [arXiv: 1505.04192] [InSPIRE].

[123] D. Baumann, D. Green, J. Meyers and B. Wallisch, Phases of new physics in the CMB, JCAP 01 (2016) 007 [arXiv: 1508.06342] [INSPIRE].

[124] C. Brust, Y. Cui and K. Sigurdson, Cosmological constraints on interacting light particles, JCAP 08 (2017) 020 [arXiv: 1703.10732] [INSPIRE].

[125] Y. Cui and R. Huo, Visualizing invisible dark matter annihilation with the CMB and matter power spectrum, arXiv:1805.06451 [INSPIRE].

[126] R.H. Cyburt, B.D. Fields, K.A. Olive and T.-H. Yeh, Big bang nucleosynthesis: 2015, Rev. Mod. Phys. 88 (2016) 015004 [arXiv: 1505.01076] [INSPIRE].

[127] R.A. Battye, R.R. Caldwell and E.P.S. Shellard, Gravitational waves from cosmic strings, in Topological defects in cosmology, (1997), pg. 11 [astro-ph/9706013] [INSPIRE].

[128] K. Saikawa and S. Shirai, Primordial gravitational waves, precisely: the role of thermodynamics in the Standard Model, JCAP 05 (2018) 035 [arXiv:1803.01038] [INSPIRE].

[129] T.L. Smith, E. Pierpaoli and M. Kamionkowski, A new cosmic microwave background constraint to primordial gravitational waves, Phys. Rev. Lett. 97 (2006) 021301 [astro-ph/0603144] [INSPIRE].

[130] S. Henrot-Versille et al., Improved constraint on the primordial gravitational-wave density using recent cosmological data and its impact on cosmic string models, Class. Quant. Grav. 32 (2015) 045003 [arXiv:1408.5299] [INSPIRE].

[131] LIGO SCIENTIFIC and VIRGO collaborations, GW170817: observation of gravitational waves from a binary neutron star inspiral, Phys. Rev. Lett. 119 (2017) 161101 [arXiv: 1710.05832] [INSPIRE].

[132] LIGO SCIENTIFIC and VIRgo collaborations, GW170608: observation of a 19-solar-mass binary black hole coalescence, Astrophys. J. 851 (2017) L35 [arXiv:1711. 05578] [INSPIRE].

[133] LIGO SCIENTIFIC and VIRGO collaborations, GW170104: observation of a 50-solar-mass binary black hole coalescence at redshift 0.2, Phys. Rev. Lett. 118 (2017) 221101 [Erratum ibid. 121 (2018) 129901] [arXiv:1706.01812] [INSPIRE].

[134] LIGO ScIENTIFIC and VIRGO collaborations, GW170817: implications for the stochastic gravitational-wave background from compact binary coalescences, Phys. Rev. Lett. 120 (2018) 091101 [arXiv: 1710.05837] [inSPIRE].

[135] LIGO Scientific and Virgo collaborations, Upper limits on the stochastic gravitational-wave background from advanced LIGO's first observing run, Phys. Rev. Lett. 118 (2017) 121101 [Erratum ibid. 119 (2017) 029901] [arXiv: 1612.02029] [INSPIRE].

[136] C.J. Moore, R.H. Cole and C.P.L. Berry, Gravitational-wave sensitivity curves, Class. Quant. Grav. 32 (2015) 015014 [arXiv: 1408.0740] [inSPIRE].

[137] X.-J. Zhu, E.J. Howell, D.G. Blair and Z.-H. Zhu, On the gravitational wave background from compact binary coalescences in the band of ground-based interferometers, Mon. Not. Roy. Astron. Soc. 431 (2013) 882 [arXiv:1209.0595] [INSPIRE]. 
[138] J. Harms, C. Mahrdt, M. Otto and M. Priess, Subtraction-noise projection in gravitational-wave detector networks, Phys. Rev. D 77 (2008) 123010 [arXiv:0803.0226] [INSPIRE].

[139] T. Regimbau, M. Evans, N. Christensen, E. Katsavounidis, B. Sathyaprakash and S. Vitale, Digging deeper: observing primordial gravitational waves below the binary black hole produced stochastic background, Phys. Rev. Lett. 118 (2017) 151105 [arXiv:1611.08943] [INSPIRE].

[140] A.C. Jenkins and M. Sakellariadou, Anisotropies in the stochastic gravitational-wave background: formalism and the cosmic string case, Phys. Rev. D 98 (2018) 063509 [arXiv: 1802.06046] [INSPIRE].

[141] M. Raidal, V. Vaskonen and H. Veermäe, Gravitational waves from primordial black hole mergers, JCAP 09 (2017) 037 [arXiv: 1707.01480] [INSPIRE].

[142] B. Carr, M. Raidal, T. Tenkanen, V. Vaskonen and H. Veermäe, Primordial black hole constraints for extended mass functions, Phys. Rev. D 96 (2017) 023514 [arXiv: 1705. 05567] [INSPIRE].

[143] B. Carr, F. Kuhnel and M. Sandstad, Primordial black holes as dark matter, Phys. Rev. D 94 (2016) 083504 [arXiv: 1607.06077] [INSPIRE].

[144] R. Smith and E. Thrane, Optimal search for an astrophysical gravitational-wave background, Phys. Rev. X 8 (2018) 021019 [arXiv: 1712.00688] [INSPIRE].

[145] P. Binetruy, A. Bohe, C. Caprini and J.-F. Dufaux, Cosmological backgrounds of gravitational waves and eLISA/NGO: phase transitions, cosmic strings and other sources, JCAP 06 (2012) 027 [arXiv:1201.0983] [INSPIRE].

[146] S. Kuroyanagi, T. Chiba and T. Takahashi, Probing the universe through the stochastic gravitational wave background, JCAP 11 (2018) 038 [arXiv:1807.00786] [INSPIRE].

[147] S.Y. Khlebnikov and I.I. Tkachev, Relic gravitational waves produced after preheating, Phys. Rev. D 56 (1997) 653 [hep-ph/9701423] [INSPIRE].

[148] R. Easther and E.A. Lim, Stochastic gravitational wave production after inflation, JCAP 04 (2006) 010 [astro-ph/0601617] [INSPIRE].

[149] R. Easther, J.T. Giblin, Jr. and E.A. Lim, Gravitational wave production at the end of inflation, Phys. Rev. Lett. 99 (2007) 221301 [astro-ph/0612294] [INSPIRE].

[150] J. García-Bellido and D.G. Figueroa, A stochastic background of gravitational waves from hybrid preheating, Phys. Rev. Lett. 98 (2007) 061302 [astro-ph/0701014] [INSPIRE].

[151] E. Witten, Cosmic separation of phases, Phys. Rev. D 30 (1984) 272 [InSPIRE].

[152] C.J. Hogan, Gravitational radiation from cosmological phase transitions, Mon. Not. Roy. Astron. Soc. 218 (1986) 629 [INSPIRE].

[153] A. Kosowsky, M.S. Turner and R. Watkins, Gravitational radiation from colliding vacuum bubbles, Phys. Rev. D 45 (1992) 4514 [InSPIRE].

[154] M. Gleiser and R. Roberts, Gravitational waves from collapsing vacuum domains, Phys. Rev. Lett. 81 (1998) 5497 [astro-ph/9807260] [INSPIRE].

[155] T. Hiramatsu, M. Kawasaki and K. Saikawa, On the estimation of gravitational wave spectrum from cosmic domain walls, JCAP 02 (2014) 031 [arXiv:1309.5001] [INSPIRE]. 
[156] C. Caprini et al., Science with the space-based interferometer eLISA. II: gravitational waves from cosmological phase transitions, JCAP 04 (2016) 001 [arXiv: 1512.06239] [INSPIRE].

[157] D.J. Weir, Gravitational waves from a first order electroweak phase transition: a brief review, Phil. Trans. Roy. Soc. Lond. A 376 (2018) 20170126 [arXiv:1705.01783] [INSPIRE].

[158] R.-G. Cai, Z. Cao, Z.-K. Guo, S.-J. Wang and T. Yang, The gravitational-wave physics, Natl. Sci. Rev. 4 (2017) 687 [arXiv:1703.00187] [INSPIRE].

[159] C. Grojean and G. Servant, Gravitational waves from phase transitions at the electroweak scale and beyond, Phys. Rev. D 75 (2007) 043507 [hep-ph/0607107] [INSPIRE].

[160] C. Caprini, R. Durrer, T. Konstandin and G. Servant, General properties of the gravitational wave spectrum from phase transitions, Phys. Rev. D 79 (2009) 083519 [arXiv:0901.1661] [INSPIRE].

[161] A. Ashoorioon and T. Konstandin, Strong electroweak phase transitions without collider traces, JHEP 07 (2009) 086 [arXiv:0904.0353] [INSPIRE].

[162] D.J.H. Chung and P. Zhou, Gravity waves as a probe of Hubble expansion rate during an electroweak scale phase transition, Phys. Rev. D 82 (2010) 024027 [arXiv:1003.2462] [INSPIRE].

[163] M. Chala, G. Nardini and I. Sobolev, Unified explanation for dark matter and electroweak baryogenesis with direct detection and gravitational wave signatures, Phys. Rev. D 94 (2016) 055006 [arXiv: 1605.08663] [INSPIRE].

[164] P. Huang, A.J. Long and L.-T. Wang, Probing the electroweak phase transition with Higgs factories and gravitational waves, Phys. Rev. D 94 (2016) 075008 [arXiv:1608.06619] [INSPIRE].

[165] A. Beniwal, M. Lewicki, J.D. Wells, M. White and A.G. Williams, Gravitational wave, collider and dark matter signals from a scalar singlet electroweak baryogenesis, JHEP 08 (2017) 108 [arXiv: 1702.06124] [INSPIRE].

[166] N. Barnaby, E. Pajer and M. Peloso, Gauge field production in axion inflation: consequences for monodromy, non-Gaussianity in the CMB and gravitational waves at interferometers, Phys. Rev. D 85 (2012) 023525 [arXiv:1110.3327] [InSPIRE].

[167] S. Kuroyanagi, T. Takahashi and S. Yokoyama, Blue-tilted tensor spectrum and thermal history of the universe, JCAP 02 (2015) 003 [arXiv:1407.4785] [INSPIRE].

[168] S. Ghosh, R. Khatri and T.S. Roy, Dark neutrino interactions make gravitational waves blue, Phys. Rev. D 97 (2018) 063529 [arXiv:1711.09929] [INSPIRE]. 